\title{
Mean-Square Convergence of Drift-Implicit One-Step Methods for Neutral Stochastic Delay Differential Equations with Jump Diffusion
}

\author{
Lin $\mathrm{Hu}^{\mathbf{1}, 2}$ and Siqing Gan \\ ${ }^{1}$ School of Mathematical Sciences and Computing Technology, Central South University, Changsha, \\ Hunan 410075, China \\ ${ }^{2}$ College of Science, Northeast Forestry University, Harbin, Heilongjiang 150040, China
}

Correspondence should be addressed to Siqing Gan, siqinggan@yahoo.com.cn

Received 1 August 2011; Accepted 11 October 2011

Academic Editor: Xiaohua Ding

Copyright (C) 2011 L. Hu and S. Gan. This is an open access article distributed under the Creative Commons Attribution License, which permits unrestricted use, distribution, and reproduction in any medium, provided the original work is properly cited.

\begin{abstract}
A class of drift-implicit one-step schemes are proposed for the neutral stochastic delay differential equations (NSDDEs) driven by Poisson processes. A general framework for mean-square convergence of the methods is provided. It is shown that under certain conditions global error estimates for a method can be inferred from estimates on its local error. The applicability of the mean-square convergence theory is illustrated by the stochastic $\theta$-methods and the balanced implicit methods. It is derived from Theorem 3.1 that the order of the mean-square convergence of both of them for NSDDEs with jumps is $1 / 2$. Numerical experiments illustrate the theoretical results. It is worth noting that the results of mean-square convergence of the stochastic $\theta$-methods and the balanced implicit methods are also new.
\end{abstract}

\section{Introduction}

In stochastic numerical analysis, the order of convergence plays a crucial role in the design of numerical algorithms. Unlike in the deterministic modelling situation, there exist in the stochastic environment different types of convergence. Both in the literature and in practice, most attention is focused on two major types of convergence, that is, strong convergence and weak convergence. There is a rich literature on this subject; we here only mention [1-4] and the references therein.

For stochastic differential equations (SDEs), Milstein [1] presented a fundamental convergence theorem which established the order of mean-square convergence of explicit 
one-step methods. The conditions of this theorem use both properties of mean and meansquare deviation of one-step approximation. The theorem showed that under certain conditions global error estimates for a method can be inferred from estimates on its local error. Buckwar [4] extended the convergence theory in [1] to stochastic functional differential equations. Recently, Zhang and Gan [5] extend the theory to neutral stochastic differential delay equations (NSDDEs). Therefore, the convergence theory in [1] and its generalization have received some attention in the case of nonjump SDEs. However, in the jump-SDE context, which is becoming increasingly important in mathematical finance [6-8], to our best knowledge, no corresponding convergence theory of numerical methods for NSDDEs with jumps has been presented in the literature. Motivated by the work of Zhang and Gan [5], our aim is to establish a relationship between the consistent order and the convergence order of the methods for the NSDDEs with jumps.

In this paper, a class of drift-implicit one-step schemes are proposed for NSDDEs driven by Poisson processes. A general framework for mean-square convergence of the methods is provided. It is shown that under certain conditions global error estimates for a method can be inferred from estimates on its local error. The applicability of the theory about mean-square convergence is illustrated by the stochastic $\theta$-methods and the balanced implicit methods. It is derived from Theorem 3.5 that the order of the mean square convergence of both of them for NSDDEs with jumps is 12. It is worth noting that the results of mean-square convergence of the stochastic $\theta$-methods and the balanced implicit methods are also new.

\section{Neutral Stochastic Delay Differential Equations with Jumps}

Let $\left(\Omega, \mathcal{F},\left\{\mathcal{F}_{t}\right\}_{t \geq 0}, \mathbb{P}\right)$ be a complete probability space with a filtration $\left\{\mathcal{F}_{t}\right\}_{t \geq 0}$ satisfying the usual conditions (i.e., it is right continuous and $\mathscr{F}_{0}$ contains all the $\mathbb{P}$-null sets). Let $W(t):=$ $\left(W_{1}(t), \ldots, W_{b}(t)\right)^{T}$ be a $b$-dimensional Wiener process, and $N(t)$ is a scalar Poisson process with intensity $\lambda(\lambda>0)$, both defined on the space $\left(\Omega, \mathcal{F},\left\{\mathcal{F}_{t}\right\}_{t \geqslant 0}, \mathbb{P}\right)$. And $|\cdot|$ is used to denote both the norm in $\mathbb{R}^{d}$ and the trace norm (F-norm) in $\mathbb{R}^{d \times b}$. Also, $C\left(\left[t_{1}, t_{2}\right] ; \mathbb{R}^{d}\right)$ is used to represent the family of continuous mappings $\psi$ from $\left[t_{1}, t_{2}\right]$ to $\mathbb{R}^{d}$. Finally, $L_{\mathscr{f}_{t}}^{p}\left(\left[t_{1}, t_{2}\right] ; \mathbb{R}^{d}\right)$ is used to denote a family of $\mathcal{F}_{t}$-measurable, $C\left(\left[t_{1}, t_{2}\right] ; \mathbb{R}^{d}\right)$-valued random variables $\psi=\{\psi(u)$ : $\left.t_{1} \leq u \leq t_{2}\right\}$ such that $\|\psi\|_{\mathbb{E}}^{p}:=\sup _{t_{1} \leq u \leq t_{2}} E|\psi(u)|^{p}<\infty$. $\mathbb{E}$ denote mathematical expectation with respect to $\mathbb{P}$.

Consider the neutral stochastic delay differential equations (NSDDEs) with Poissondriven jumps

$$
\begin{aligned}
\mathrm{d}[x(t)-G(x(t-\tau))]= & f\left(x\left(t^{-}\right), x\left(t^{-}-\tau\right)\right) \mathrm{d} t+g\left(x\left(t^{-}\right), x\left(t^{-}-\tau\right)\right) \mathrm{d} W(t) \\
& +u\left(x\left(t^{-}\right), x\left(t^{-}-\tau\right)\right) \mathrm{d} N(t), \quad t \in[0, T],
\end{aligned}
$$

with initial data

$$
x(t)=\psi(t), \quad t \in[-\tau, 0],
$$

where $\psi(t) \in L_{\mathcal{q}_{0}}^{2}\left([-\tau, 0] ; \mathbb{R}^{d}\right)$. Here, $\tau>0$ is a constant, $x\left(t^{-}\right)$denotes $\lim _{s \rightarrow t^{-}} x(s), f: \mathbb{R}^{d} \times$ $\mathbb{R}^{d} \rightarrow \mathbb{R}^{d}, g: \mathbb{R}^{d} \times \mathbb{R}^{d} \rightarrow \mathbb{R}^{d \times b}, u: \mathbb{R}^{d} \times \mathbb{R}^{d} \rightarrow \mathbb{R}^{d}$, and $G: \mathbb{R}^{d} \rightarrow \mathbb{R}^{d}$ 
By the definition of Itô-interpreted stochastic differential equations, the integral version of (2.1) is expressed as follows:

$$
\begin{aligned}
x(t)= & G(x(t-\tau))+x(0)-G(x(-\tau))+\int_{0}^{t} f\left(x\left(s^{-}\right), x\left(s^{-}-\tau\right)\right) d s \\
& +\int_{0}^{t} g\left(x\left(s^{-}\right), x\left(s^{-}-\tau\right)\right) d W(s)+\int_{0}^{t} u\left(x\left(s^{-}\right), x\left(s^{-}-\tau\right)\right) d N(s), \quad t>0 .
\end{aligned}
$$

Definition 2.1 (see [9]). An $\mathbb{R}^{d}$-valued stochastic process $x(t)$ on $-\tau \leq t \leq T$ is called a solution to (2.1) with initial data (2.2) if it has the following properties:

(i) $\{x(t)\}_{0 \leq t \leq T}$ is continuous and $\mathcal{F}_{t}$-adapted;

(ii) $\{f(x(t), x(t-\tau))\} \in \mathcal{L}^{1}\left([0, T] ; \mathbb{R}^{d}\right),\{g(x(t), x(t-\tau))\} \in \mathcal{L}^{2}\left([0, T] ; \mathbb{R}^{d \times b}\right)$, $\{u(x(t), x(t-\tau))\} \in \mathcal{L}^{1}\left([0, T] ; \mathbb{R}^{d}\right) ;$

(iii) $x(t)=\psi(t),-\tau \leq t \leq 0$, and (2.3) holds for every $t \in[0, T]$ with probability 1 , where $\mathcal{L}^{p}\left([0, T] ; \mathbb{R}^{d}\right)$ denotes the family of Borel measurable functions $\varpi:[0, T] \rightarrow \mathbb{R}^{d}$ such that $\int_{0}^{T}|\varpi(t)|^{p} d t<\infty$ a.s.

A solution $\{x(t)\}$ is said to be unique if any other solution $\{\bar{x}(t)\}$ is indistinguishable from $\{x(t)\}$, that is,

$$
\mathbb{P}\{x(t)=\bar{x}(t) \forall t \in[0 T]\}=1 .
$$

In order to guarantee the existence and uniqueness of the solution, we impose the following hypothesis.

Assumption 1 (global Lipschitz condition). There exists a positive constant $K$ such that for all $x_{1}, x_{2}, y_{1}, y_{2} \in \mathbb{R}^{d}$,

$$
\left|v\left(x_{1}, y_{1}\right)-v\left(x_{2}, y_{2}\right)\right|^{2} \leqslant K\left(\left|x_{1}-x_{2}\right|^{2}+\left|y_{1}-y_{2}\right|^{2}\right), \quad v=f, g \text { or } u
$$

Assumption 2 (linear growth condition). There exists a positive constant $L$ such that for all $x, y \in \mathbb{R}^{d}$,

$$
|v(x, y)|^{2} \leqslant L\left(1+|x|^{2}+|y|^{2}\right), \quad v=f, g \text { or } u \text {. }
$$

Assumption 3. There is a constant $\eta \in(0,1)$ such that for all $x_{1}, x_{2} \in \mathbb{R}^{d}$

$$
\left|G\left(x_{1}\right)-G\left(x_{2}\right)\right| \leq \eta\left|x_{1}-x_{2}\right|
$$

Remark 2.2. In this paper, we always assume that $\eta \neq 0$. Otherwise, the system (2.1) reduces to the stochastic delay differential equations with jumps.

We use $J_{1}, J_{2}, \ldots$ to denote the constants which are independent of the stepsize $h$. 
Lemma 2.3. If Assumptions 1-3 hold, then (2.1) has a unique strong solution $x(t)$ on $t \geq-\tau$, and the solution of (2.1) satisfies

$$
\mathbb{E}\left(\sup _{-\tau \leq t \leq T}|x(t)|^{2}\right) \leq J_{1}
$$

where $J_{1}$ is a positive constant which depends on constants $T, \eta, L$, and initial function $\psi(t)$.

It is not hard to prove Lemma 2.3 in a similar way as the proof of Theorems 6.2.2 and 6.4 .5 in [9].

Lemma 2.4. Let Assumptions 2 and 3 hold. Assume that the initial function $\psi(t)$ is uniformly Lipschitz $L^{2}$-continuous, that is, there is a positive constant $\bar{H}$ such that

$$
\mathbb{E}\left|\psi\left(u_{1}\right)-\psi\left(u_{2}\right)\right|^{2} \leq \bar{H}\left(u_{1}-u_{2}\right), \quad \text { if }-\tau \leq u_{2}<u_{1} \leq 0,
$$

then

$$
\mathbb{E}\left|x\left(t_{1}\right)-x\left(t_{2}\right)\right|^{2} \leq \widehat{H}\left(t_{1}-t_{2}\right),
$$

for all $0 \leq t_{1}<t_{2} \leq T$ with $t_{1}-s \tau \in[-\tau, 0], t_{2}-s \tau \in[-\tau, 0]$, where the constant $\widehat{H}$ depends on constants $\bar{H}, T$, initial function $\psi(t)$, and positive integer $s$.

Lemma 2.4 is a modified version of [10, Lemma 2.1]. In a similar way, it is not hard to obtain the estimate (2.10). have

In this paper, we will use the following inequality. For any $a, c>0$ and $0<\alpha<1$, we

$$
(a+c)^{2} \leq \frac{a^{2}}{\alpha}+\frac{c^{2}}{1-\alpha} .
$$

\section{Implicit One-Step Schemes}

Define a mesh with uniform step $h$ which satisfies $\tau=m h$ for an integer number $m$ (for convenience, we assume that $m \geqslant 2$ ), and suppose that $q$ is a positive integer with $q=T / h$. Let $t_{n}=n h, n=0,1, \ldots, q$. The drift-implicit one-step methods for the simulation of the solution $x(t)$ of (2.1) are defined as follows:

$$
\begin{aligned}
& Y_{n+1}= G\left(Y_{n+1-m}\right)-G\left(Y_{n-m}\right)+Y_{n} \\
&+\Phi_{f}\left(h, Y_{n}, Y_{n+1}, Y_{n-m}, Y_{n+1-m}, \Delta W_{n}, \Delta N_{n}\right) \\
&+\Phi_{g}\left(h, Y_{n}, Y_{n-m}, \Delta W_{n}, \Delta N_{n}\right)+\Phi_{u}\left(h, Y_{n}, Y_{n-m}, \Delta W_{n}, \Delta N_{n}\right), \\
& n=0,1, \ldots, q-1,
\end{aligned}
$$


where $Y_{n}$ is an approximation of the exact solution $x\left(t_{n}\right)$, and $\Phi_{v}(v=f, g$, and $u)$ are increment functions. $\Delta W_{n}=W\left(t_{n+1}\right)-W\left(t_{n}\right), \Delta N_{n}=N\left(t_{n+1}\right)-N\left(t_{n}\right)$, and $\Delta W_{n}$ is independent of $\Delta N_{n} . Y_{n-m}=\psi\left(t_{n}-\tau\right)$ when $n-m \leqslant 0$.

Remark 3.1. Now, we discuss the solvability of (3.1). If increment function $\Phi_{f}$ does not depend on $Y_{n+1}$, it is not difficult to find that the approximations $Y_{n}$ can be computed iteratively. If $\Phi_{f}$ depends on $Y_{n+1}$, in order to guarantee the existence and uniqueness of a solution, the general approach is to assume Lipschitz continuity of $\Phi_{f}$ with respect to $Y_{n+1}$ with the Lipschitz constant less than 1, and then to apply Banach's contraction mapping principle [4].

We denote by $\bar{Y}\left(t_{n+1}\right)$ the value that is obtained when the exact solution values are inserted into the right-hand side of (3.1), that is,

$$
\begin{aligned}
& \bar{Y}\left(t_{n+1}\right)= G\left(x\left(t_{n+1}-\tau\right)\right)-G\left(x\left(t_{n}-\tau\right)\right)+x\left(t_{n}\right) \\
&+\Phi_{f}\left(h, x\left(t_{n}\right), x\left(t_{n+1}\right), x\left(t_{n}-\tau\right), x\left(t_{n+1}-\tau\right), \Delta W_{n}, \Delta N_{n}\right) \\
&+\Phi_{g}\left(h, x\left(t_{n}\right), x\left(t_{n}-\tau\right), \Delta W_{n}, \Delta N_{n}\right)+\Phi_{u}\left(h, x\left(t_{n}\right), x\left(t_{n}-\tau\right), \Delta W_{n}, \Delta N_{n}\right), \\
& n=0,1, \ldots, q-1 .
\end{aligned}
$$

We introduce the following definitions, which are presented in the literature, see $[1,3]$, for example.

Definition 3.2. The local error of method (3.1) is the sequence of random variables

$$
\delta_{n+1}:=x\left(t_{n+1}\right)-\bar{Y}\left(t_{n+1}\right), \quad n=0,1, \ldots, q-1 .
$$

The global error of method (3.1) is the sequence of random variables

$$
\varepsilon_{n}:=x\left(t_{n}\right)-Y_{n}, \quad n=0,1, \ldots, q .
$$

Definition 3.3. The numerical method (3.1) is said to be consistent with order $p_{1}$ in the mean and with order $p_{2}$ in the mean square sense if the following estimates hold with $p_{2} \geqslant 1 / 2$ and $p_{1} \geqslant p_{2}+1 / 2$ :

$$
\begin{gathered}
\max _{0 \leq n \leq q-1}\left\|\mathbb{E}\left(\delta_{n+1} \mid \mathcal{F}_{t_{n}}\right)\right\|_{L_{2}} \leq H_{0} h^{p_{1}}, \quad \text { as } h \longrightarrow 0, \\
\max _{0 \leq n \leq q-1}\left\|\delta_{n+1}\right\|_{L_{2}} \leq H_{1} h^{p_{2}}, \quad \text { as } h \longrightarrow 0
\end{gathered}
$$

where the constants $H_{0}$ and $H_{1}$ do not depend on $h$ but may depend on $T$ and the initial data. Here, $\|z\|_{L_{2}}:=\left(\mathbb{E}|z|^{2}\right)^{1 / 2}$. 
Definition 3.4. The numerical method (3.1) is said to be convergent with order $p$ in the mean square sense, on the mesh points, if the following estimate holds:

$$
\max _{1 \leq n \leq q}\left\|\varepsilon_{n}\right\|_{L_{2}} \leq H_{2} h^{p}, \quad \text { as } h \longrightarrow 0,
$$

where the constant $H_{2}$ does not depend on $h$ but may depend on $T$ and the initial data.

In order to obtain the main result, the following properties of the increment functions are required. There exist positive constants $L_{f}$ such that for $x_{i}, y_{i} \in \mathbb{R}^{d}(i=1,2,3,4)$,

$$
\left|\Phi_{f}\left(h, x_{1}, x_{2}, x_{3}, x_{4}, \Delta W_{n}, \Delta N_{n}\right)-\Phi_{f}\left(h, y_{1}, y_{2}, y_{3}, y_{4}, \Delta W_{n}, \Delta N_{n}\right)\right| \leq h L_{f} \sum_{i=1}^{4}\left|x_{i}-y_{i}\right|
$$

and there exist the positive constants $L_{g}, L_{u}, L_{\bar{u}}$ such that for all $\mathcal{F}_{t_{n}}$-measurable random variables $x_{1}, x_{3}, y_{1}, y_{3} \in \mathbb{R}^{d}$,

$$
\begin{gathered}
\mathbb{E}\left|\Phi_{g}\left(h, x_{1}, x_{3}, \Delta W_{n}, \Delta N_{n}\right)-\Phi_{g}\left(h, y_{1}, y_{3}, \Delta W_{n}, \Delta N_{n}\right)\right|^{2} \\
\leq h L_{g}\left[\mathbb{E}\left|x_{1}-y_{1}\right|^{2}+\mathbb{E}\left|x_{3}-y_{3}\right|^{2}\right], \\
\mathbb{E}\left|\Phi_{u}\left(h, x_{1}, x_{3}, \Delta W_{n}, \Delta N_{n}\right)-\Phi_{u}\left(h, y_{1}, y_{3}, \Delta W_{n}, \Delta N_{n}\right)\right|^{2} \\
\leq h L_{u}\left[\mathbb{E}\left|x_{1}-y_{1}\right|^{2}+\mathbb{E}\left|x_{3}-y_{3}\right|^{2}\right], \\
\mathbb{E}\left(\Phi_{g}\left(h, x_{1}, x_{3}, \Delta W_{n}, \Delta N_{n}\right)-\Phi_{g}\left(h, y_{1}, y_{3}, \Delta W_{n}, \Delta N_{n}\right) \mid \mathscr{F}_{t_{n}}\right)=0, \\
\left|\mathbb{E}\left[\Phi_{u}\left(h, x_{1}, x_{3}, \Delta W_{n}, \Delta N_{n}\right)-\Phi_{u}\left(h, y_{1}, y_{3}, \Delta W_{n}, \Delta N_{n}\right) \mid F_{t_{n}}\right]\right| \\
\leq h L_{\bar{u}}\left[\left|x_{1}-y_{1}\right|+\left|x_{3}-y_{3}\right|\right] .
\end{gathered}
$$

Now, we state our result on the convergence of the one-step method (3.1).

Theorem 3.5. Suppose that Assumption 3 and the conditions (3.7)-(3.11) hold. Assume that the one-step method (3.1) is consistent with order $p_{1}$ in the mean and order $p_{2}$ in the mean square sense, then the method (3.1) is convergent with order $p=p_{2}-1 / 2$ in the mean square sense.

Proof. By (3.4), we have

$$
\varepsilon_{n+1}=\widehat{\varepsilon}_{n+1}+\left[G\left(x\left(t_{n+1}-\tau\right)\right)-G\left(Y_{n+1-m}\right)\right],
$$

where $\widehat{\varepsilon}_{n+1}$ is defined as follows:

$$
\widehat{\varepsilon}_{n+1}=x\left(t_{n+1}\right)-G\left(x\left(t_{n+1}-\tau\right)\right)-\left[Y_{n+1}-G\left(Y_{n+1-m}\right)\right] .
$$


Squaring and taking expectation on both sides of (3.12), using Assumption 3 and (2.11), we have

$$
\begin{aligned}
\mathbb{E}\left|\varepsilon_{n+1}\right|^{2} & \leq \frac{1}{1-\eta} \mathbb{E}\left|\widehat{\varepsilon}_{n+1}\right|^{2}+\frac{1}{\eta} \mathbb{E}\left|G\left(x\left(t_{n+1}-\tau\right)\right)-G\left(Y_{n+1-m}\right)\right|^{2} \\
& \leq \frac{1}{1-\eta} \mathbb{E}\left|\widehat{\varepsilon}_{n+1}\right|^{2}+\eta \mathbb{E}\left|x\left(t_{n+1}-\tau\right)-Y_{n+1-m}\right|^{2} \\
& =\frac{1}{1-\eta} \mathbb{E}\left|\widehat{\varepsilon}_{n+1}\right|^{2}+\eta \mathbb{E}\left|\varepsilon_{n+1-m}\right|^{2} .
\end{aligned}
$$

It follows from (3.1), (3.2), and (3.13) that

$$
\begin{aligned}
\widehat{\varepsilon}_{n+1}= & x\left(t_{n+1}\right)-G\left(x\left(t_{n+1}-\tau\right)\right)-\bar{Y}\left(t_{n+1}\right)+\bar{Y}\left(t_{n+1}\right)-\left[Y_{n+1}-G\left(Y_{n+1-m}\right)\right] \\
= & x\left(t_{n+1}\right)-\bar{Y}\left(t_{n+1}\right)+x\left(t_{n}\right)-G\left(x\left(t_{n}-\tau\right)\right) \\
& +\Phi_{f}\left(h, x\left(t_{n}\right), x\left(t_{n+1}\right), x\left(t_{n}-\tau\right), x\left(t_{n+1}-\tau\right), \Delta W_{n}, \Delta N_{n}\right) \\
& +\Phi_{g}\left(h, x\left(t_{n}\right), x\left(t_{n}-\tau\right), \Delta W_{n}, \Delta N_{n}\right)+\Phi_{u}\left(h, x\left(t_{n}\right), x\left(t_{n}-\tau\right), \Delta W_{n}, \Delta N_{n}\right) \\
& -Y_{n}+G\left(Y_{n-m}\right)-\Phi_{f}\left(h, Y_{n}, Y_{n+1}, Y_{n-m}, Y_{n+1-m}, \Delta W_{n}, \Delta N_{n}\right) \\
& -\Phi_{g}\left(h, Y_{n}, Y_{n-m}, \Delta W_{n}, \Delta N_{n}\right)-\Phi_{u}\left(h, Y_{n}, Y_{n-m}, \Delta W_{n}, \Delta N_{n}\right) \\
= & \delta_{n+1}+\widehat{\varepsilon}_{n}+R_{n},
\end{aligned}
$$

where

$$
\begin{aligned}
R_{n}= & \Phi_{f}\left(h, x\left(t_{n}\right), x\left(t_{n+1}\right), x\left(t_{n}-\tau\right), x\left(t_{n+1}-\tau\right), \Delta W_{n}, \Delta N_{n}\right) \\
& -\Phi_{f}\left(h, Y_{n}, Y_{n+1}, Y_{n-m}, Y_{n+1-m}, \Delta W_{n}, \Delta N_{n}\right) \\
& +\Phi_{g}\left(h, x\left(t_{n}\right), x\left(t_{n}-\tau\right), \Delta W_{n}, \Delta N_{n}\right)-\Phi_{g}\left(h, Y_{n}, Y_{n-m}, \Delta W_{n}, \Delta N_{n}\right) \\
& +\Phi_{u}\left(h, x\left(t_{n}\right), x\left(t_{n}-\tau\right), \Delta W_{n}, \Delta N_{n}\right)-\Phi_{u}\left(h, Y_{n}, Y_{n-m}, \Delta W_{n}, \Delta N_{n}\right) .
\end{aligned}
$$

Squaring and taking expectation on both sides of (3.15) yields

$$
\mathbb{E}\left|\widehat{\varepsilon}_{n+1}\right|^{2} \leq \mathbb{E}\left|\widehat{\varepsilon}_{n}\right|^{2}+2 \mathbb{E}\left|\delta_{n+1}\right|^{2}+2 \mathbb{E}\left|R_{n}\right|^{2}+2 \mathbb{E}\left\langle\delta_{n+1}, \widehat{\varepsilon}_{n}\right\rangle+2 \mathbb{E}\left\langle\widehat{\varepsilon}_{n}, R_{n}\right\rangle
$$

We will now estimate the separate terms in (3.17) individually. Without loss of generality, we can assume that $0<h<1$. We notice that the method (3.1) is consistent with order $p_{2}$ in the mean square sense; thus, there exists a constant $J_{2}$ such that

$$
\mathbb{E}\left|\delta_{n+1}\right|^{2} \leq J_{2} h^{2 p_{2}}
$$


By (3.7)-(3.9), we obtain

$$
\begin{aligned}
\mathbb{E}\left|R_{n}\right|^{2} \leq & 3 \mathbb{E} \mid \Phi_{f}\left(h, x\left(t_{n}\right), x\left(t_{n+1}\right), x\left(t_{n}-\tau\right), x\left(t_{n+1}-\tau\right), \Delta W_{n}, \Delta N_{n}\right) \\
& -\left.\Phi_{f}\left(h, Y_{n}, Y_{n+1}, Y_{n-m}, Y_{n+1-m}, \Delta W_{n}, \Delta N_{n}\right)\right|^{2} \\
& +3 \mathbb{E}\left|\Phi_{g}\left(h, x\left(t_{n}\right), x\left(t_{n}-\tau\right), \Delta W_{n}, \Delta N_{n}\right)-\Phi_{g}\left(h, Y_{n}, Y_{n-m}, \Delta W_{n}, \Delta N_{n}\right)\right|^{2} \\
& +3 \mathbb{E}\left|\Phi_{u}\left(h, x\left(t_{n}\right), x\left(t_{n}-\tau\right), \Delta W_{n}, \Delta N_{n}\right)-\Phi_{u}\left(h, Y_{n}, Y_{n-m}, \Delta W_{n}, \Delta N_{n}\right)\right|^{2} \\
\leq & 3 h^{2} L_{f}^{2} \mathbb{E}\left(\left|x\left(t_{n}\right)-Y_{n}\right|+\left|x\left(t_{n+1}\right)-Y_{n+1}\right|+\left|x\left(t_{n}-\tau\right)-Y_{n-m}\right|\right. \\
& \left.\quad+\left|x\left(t_{n+1}-\tau\right)-Y_{n+1-m}\right|\right)^{2}+3 h L_{g}\left(\mathbb{E}\left|x\left(t_{n}\right)-Y_{n}\right|^{2}+\mathbb{E}\left|x\left(t_{n}-\tau\right)-Y_{n-m}\right|^{2}\right) \\
& +3 h L_{u}\left(\mathbb{E}\left|x\left(t_{n}\right)-Y_{n}\right|^{2}+\mathbb{E}\left|x\left(t_{n}-\tau\right)-Y_{n-m}\right|^{2}\right) \\
\leq & \left(12 h^{2} L_{f}^{2}+3 h L_{g}+3 h L_{u}\right)\left(\mathbb{E}\left|x\left(t_{n}\right)-Y_{n}\right|^{2}+\mathbb{E}\left|x\left(t_{n}-\tau\right)-Y_{n-m}\right|^{2}\right) \\
& +12 h^{2} L_{f}^{2}\left(\mathbb{E}\left|x\left(t_{n+1}\right)-Y_{n+1}\right|^{2}+\mathbb{E}\left|x\left(t_{n+1}-\tau\right)-Y_{n+1-m}\right|^{2}\right) \\
= & \left(12 h^{2} L_{f}^{2}+3 h L_{g}+3 h L_{u}\right)\left(\mathbb{E}\left|\varepsilon_{n}\right|^{2}+\mathbb{E}\left|\varepsilon_{n-m}\right|^{2}\right)+12 h^{2} L_{f}^{2}\left(\mathbb{E}\left|\varepsilon_{n+1}\right|^{2}+\mathbb{E}\left|\varepsilon_{n+1-m}\right|^{2}\right),
\end{aligned}
$$

which, by the inequality (3.14), yields

$$
\begin{aligned}
\mathbb{E}\left|R_{n}\right|^{2} \leq & \left(12 h^{2} L_{f}^{2}+3 h L_{g}+3 h L_{u}\right)\left(\frac{1}{1-\eta} \mathbb{E}\left|\widehat{\varepsilon}_{n}\right|^{2}+\eta \mathbb{E}\left|\varepsilon_{n-m}\right|^{2}\right) \\
& +\left(12 h^{2} L_{f}^{2}+3 h L_{g}+3 h L_{u}\right) \mathbb{E}\left|\varepsilon_{n-m}\right|^{2} \\
& +12 h^{2} L_{f}^{2}\left(\frac{1}{1-\eta} \mathbb{E}\left|\widehat{\varepsilon}_{n+1}\right|^{2}+\eta \mathbb{E}\left|\varepsilon_{n+1-m}\right|^{2}\right)+12 h^{2} L_{f}^{2} \mathbb{E}\left|\varepsilon_{n+1-m}\right|^{2} \\
\leq & \left(12 h^{2} L_{f}^{2}+3 h L_{g}+3 h L_{u}\right)\left(\mathbb{E}\left|\widehat{\varepsilon}_{n}\right|^{2}+\mathbb{E}\left|\varepsilon_{n-m}\right|^{2}\right) \max \left\{\frac{1}{1-\eta}, 1+\eta\right\} \\
& +12 h^{2} L_{f}^{2}\left(\mathbb{E}\left|\widehat{\varepsilon}_{n+1}\right|^{2}+\mathbb{E}\left|\varepsilon_{n+1-m}\right|^{2}\right) \max \left\{\frac{1}{1-\eta}, 1+\eta\right\} \\
\leq & \left(12 T L_{f}^{2}+3 L_{g}+3 L_{u}\right) h\left(\mathbb{E}\left|\widehat{\varepsilon}_{n}\right|^{2}+\mathbb{E}\left|\varepsilon_{n-m}\right|^{2}+\mathbb{E}\left|\widehat{\varepsilon}_{n+1}\right|^{2}\right. \\
+ & \left.+\mathbb{E}\left|\varepsilon_{n+1-m}\right|^{2}\right) \max \left\{\frac{1}{1-\eta}, 1+\eta\right\} \\
= & J_{3} h\left(\mathbb{E}\left|\widehat{\varepsilon}_{n}\right|^{2}+\mathbb{E}\left|\varepsilon_{n-m}\right|^{2}+\mathbb{E}\left|\widehat{\varepsilon}_{n+1}\right|^{2}+\mathbb{E}\left|\varepsilon_{\mathrm{n}+1-m}\right|^{2}\right),
\end{aligned}
$$

where $J_{3}=\left(12 T L_{f}^{2}+3 L_{g}+3 L_{u}\right) \max \{1 / 1-\eta, 1+\eta\}$. 
Since method (3.1) is consistent with order $p_{1}$ in the mean square, there exists a constant $J_{4}$ such that

$$
\left(\mathbb{E}\left|\mathbb{E}\left(\delta_{n+1} \mid \mathcal{F}_{t_{n}}\right)\right|^{2}\right)^{1 / 2} \leq J_{4} h^{p_{1}}
$$

Noticing that $\widehat{\varepsilon}_{n}$ is $\mathcal{F}_{t_{n}}$-measurable and by (3.21), we arrive at

$$
\begin{aligned}
2 \mathbb{E}\left(\left\langle\widehat{\varepsilon}_{n}, \delta_{n+1}\right\rangle\right) & =2 \mathbb{E}\left[\mathbb{E}\left(\left\langle\widehat{\varepsilon}_{n}, \delta_{n+1}\right\rangle \mid \mathcal{F}_{t_{n}}\right)\right] \\
& \leq 2 \mathbb{E}\left|\mathbb{E}\left(\left\langle\widehat{\varepsilon}_{n}, \delta_{n+1}\right\rangle \mid \mathcal{F}_{t_{n}}\right)\right|=2 \mathbb{E}\left|\left\langle\widehat{\varepsilon}_{n}, \mathbb{E}\left(\delta_{n+1} \mid \mathcal{F}_{t_{n}}\right)\right\rangle\right| \\
& \leq 2\left(h \mathbb{E}\left|\widehat{\varepsilon}_{n}\right|^{2}\right)^{1 / 2}\left(h^{-1} \mathbb{E}\left|\mathbb{E}\left(\delta_{n+1} \mid \mathcal{F}_{t_{n}}\right)\right|^{2}\right)^{1 / 2} \\
& \leq h \mathbb{E}\left|\widehat{\varepsilon}_{n}\right|^{2}+h^{-1} \mathbb{E}\left|\mathbb{E}\left(\delta_{n+1} \mid \mathcal{F}_{t_{n}}\right)\right|^{2} \\
& \leq h \mathbb{E}\left|\widehat{\varepsilon}_{n}\right|^{2}+\left(J_{4}\right)^{2} h^{2 p_{1}-1} \\
& =h \mathbb{E}\left|\widehat{\varepsilon}_{n}\right|^{2}+J_{5} h^{2 p_{1}-1}
\end{aligned}
$$

where $J_{5}=\left(J_{4}\right)^{2}$. Applying the inequality $|\mathbb{E}(x \mid \mathcal{F})|^{2} \leq \mathbb{E}\left(|x|^{2} \mid \mathcal{F}\right)$, (3.7), (3.10), and (3.11) yields

$$
\begin{aligned}
& \left|\mathbb{E}\left(R_{n} \mid \mathcal{F}_{t_{n}}\right)\right|^{2}=\mid \mathbb{E}\left[\Phi_{f}\left(h, x\left(t_{n}\right), x\left(t_{n+1}\right), x\left(t_{n}-\tau\right), x\left(t_{n+1}-\tau\right), \Delta W_{n}, \Delta N_{n}\right)\right. \\
& \text { - } \Phi_{f}\left(h, Y_{n}, Y_{n+1}, Y_{n-m}, Y_{n+1-m}, \Delta W_{n}, \Delta N_{n}\right) \\
& +\Phi_{g}\left(h, x\left(t_{n}\right), x\left(t_{n}-\tau\right), \Delta W_{n}, \Delta N_{n}\right)-\Phi_{g}\left(h, Y_{n}, Y_{n-m}, \Delta W_{n}, \Delta N_{n}\right) \\
& \left.+\Phi_{u}\left(h, x\left(t_{n}\right), x\left(t_{n}-\tau\right), \Delta W_{n}, \Delta N_{n}\right)-\Phi_{u}\left(h, Y_{n}, Y_{n-m}, \Delta W_{n}, \Delta N_{n}\right) \mid \mathcal{F}_{t_{n}}\right]\left.\right|^{2} \\
& =\mid \mathbb{E}\left[\Phi_{f}\left(h, x\left(t_{n}\right), x\left(t_{n+1}\right), x\left(t_{n}-\tau\right), x\left(t_{n+1}-\tau\right), \Delta W_{n}, \Delta N_{n}\right)\right. \\
& \left.-\Phi_{f}\left(h, Y_{n}, Y_{n+1}, Y_{n-m}, Y_{n+1-m}, \Delta W_{n}, \Delta N_{n}\right) \mid \mathcal{F}_{t_{n}}\right] \\
& +\left.\mathbb{E}\left[\Phi_{u}\left(h, x\left(t_{n}\right), x\left(t_{n}-\tau\right), \Delta W_{n}, \Delta N_{n}\right)-\Phi_{u}\left(h, Y_{n}, Y_{n-m}, \Delta W_{n}, \Delta N_{n}\right) \mid \mathcal{F}_{t_{n}}\right]\right|^{2} \\
& \leq 2 \mathbb{E}\left[\mid \Phi_{f}\left(h, x\left(t_{n}\right), x\left(t_{n+1}\right), x\left(t_{n}-\tau\right), x\left(t_{n+1}-\tau\right), \Delta W_{n}, \Delta N_{n}\right)\right. \\
& \left.-\left.\Phi_{f}\left(h, Y_{n}, Y_{n+1}, Y_{n-m}, Y_{n+1-m}, \Delta W_{n}, \Delta N_{n}\right)\right|^{2} \mid \mathcal{F}_{t_{n}}\right] \\
& +2\left|\mathbb{E}\left[\Phi_{u}\left(h, x\left(t_{n}\right), x\left(t_{n}-\tau\right), \Delta W_{n}, \Delta N_{n}\right)-\Phi_{u}\left(h, Y_{n}, Y_{n-m}, \Delta W_{n}, \Delta N_{n}\right) \mid \mathcal{F}_{t_{n}}\right]\right|^{2} \\
& \leq 2 h^{2}\left(L_{f}\right)^{2} \mathbb{E}\left[\left(\left|x\left(t_{n}\right)-Y_{n}\right|+\left|x\left(t_{n+1}\right)-Y_{n+1}\right|+\left|x\left(t_{n}-\tau\right)-Y_{n-m}\right|\right.\right. \\
& \left.\left.+\left|x\left(t_{n+1}-\tau\right)-Y_{n+1-m}\right|\right)^{2} \mid \mathcal{F}_{t_{n}}\right] \\
& +2 h^{2}\left(L_{\bar{u}}\right)^{2}\left[\left|x\left(t_{n}\right)-Y_{n}\right|+\left|x\left(t_{n}-\tau\right)-Y_{n-m}\right|\right]^{2} \\
& \leq 8 h^{2}\left(L_{f}\right)^{2}\left[\left|\varepsilon_{n}\right|^{2}+\mathbb{E}\left(\left|\varepsilon_{n+1}\right|^{2} \mid \boldsymbol{F}_{t_{n}}\right)+\left|\varepsilon_{n-m}\right|^{2}+\left|\varepsilon_{n+1-m}\right|^{2}\right] \\
& +4 h^{2}\left(L_{\bar{u}}\right)^{2}\left[\left|\varepsilon_{n}\right|^{2}+\left|\varepsilon_{n-m}\right|^{2}\right]
\end{aligned}
$$




$$
\begin{aligned}
= & {\left[8 h^{2}\left(L_{f}\right)^{2}+4 h^{2}\left(L_{\bar{u}}\right)^{2}\right]\left|\varepsilon_{n}\right|^{2}+8 h^{2}\left(L_{f}\right)^{2} \mathbb{E}\left(\left|\varepsilon_{n+1}\right|^{2} \mid \mathcal{F}_{t_{n}}\right) } \\
& +\left[8 h^{2}\left(L_{f}\right)^{2}+4 h^{2}\left(L_{\bar{u}}\right)^{2}\right]\left|\varepsilon_{n-m}\right|^{2}+8 h^{2}\left(L_{f}\right)^{2}\left|\varepsilon_{n+1-m}\right|^{2} .
\end{aligned}
$$

Here, the fact used has been that $x\left(t_{n}\right), x\left(t_{n}-\tau\right), x\left(t_{n+1}-\tau\right), Y_{n}, Y_{n-m}, Y_{n+1-m}$ are $\mathcal{F}_{t_{n}}{ }^{-}$ measurable. Using (3.14) and (3.23), we arrive at

$$
\begin{aligned}
& 2 \mathbb{E}\left(\left\langle\widehat{\varepsilon}_{n}, R_{n}\right\rangle\right)=2 \mathbb{E}\left[\mathbb{E}\left(\left\langle\widehat{\varepsilon}_{n}, R_{n}\right\rangle \mid \mathcal{F}_{t_{n}}\right)\right] \\
& =2\left[\mathbb{E}\left\langle\widehat{\varepsilon}_{n}, \mathbb{E}\left(R_{n} \mid \mathcal{F}_{t_{n}}\right)\right\rangle\right] \leq 2 \mathbb{E}\left[\left|\widehat{\varepsilon}_{n}\right| \cdot\left|\mathbb{E}\left(R_{n} \mid \mathcal{F}_{t_{n}}\right)\right|\right] \\
& \leq 2\left(h \mathbb{E}\left|\widehat{\varepsilon}_{n}\right|^{2}\right)^{1 / 2}\left(h^{-1} \mathbb{E} \mid \mathbb{E}\left(R_{n} \mid \mathcal{F}_{t_{n}}\right)^{2}\right)^{1 / 2} \\
& \leq h \mathbb{E}\left|\widehat{\varepsilon}_{n}\right|^{2}+h^{-1} \mathbb{E}\left|\mathbb{E}\left(R_{n} \mid \mathcal{F}_{t_{n}}\right)\right|^{2} \\
& \leq h \mathbb{E}\left|\widehat{\varepsilon}_{n}\right|^{2}+\left[8 h\left(L_{f}\right)^{2}+4 h\left(L_{\bar{u}}\right)^{2}\right] \mathbb{E}\left|\varepsilon_{n}\right|^{2}+8 h\left(L_{f}\right)^{2} \mathbb{E}\left|\varepsilon_{n+1}\right|^{2} \\
& +\left[8 h\left(L_{f}\right)^{2}+4 h\left(L_{\bar{u}}\right)^{2}\right] \mathbb{E}\left|\varepsilon_{n-m}\right|^{2}+8 h\left(L_{f}\right)^{2} \mathbb{E}\left|\varepsilon_{n+1-m}\right|^{2} \\
& \leq h \mathbb{E}\left|\widehat{\varepsilon}_{n}\right|^{2}+\left[8 h\left(L_{f}\right)^{2}+4 h\left(L_{\bar{u}}\right)^{2}\right]\left[\frac{1}{1-\eta} \mathbb{E}\left|\widehat{\varepsilon}_{n}\right|^{2}+\eta \mathbb{E}\left|\varepsilon_{n-m}\right|^{2}\right] \\
& +8 h\left(L_{f}\right)^{2}\left[\frac{1}{1-\eta} \mathbb{E}\left|\widehat{\varepsilon}_{n+1}\right|^{2}+\eta \mathbb{E}\left|\varepsilon_{n+1-m}\right|^{2}\right] \\
& +\left[8 h\left(L_{f}\right)^{2}+4 h\left(L_{\bar{u}}\right)^{2}\right] \mathbb{E}\left|\varepsilon_{n-m}\right|^{2}+8 h\left(L_{f}\right)^{2} \mathbb{E}\left|\varepsilon_{n+1-m}\right|^{2} \\
& =\left[1+\left(8\left(L_{f}\right)^{2}+4\left(L_{\bar{u}}\right)^{2}\right) \frac{1}{1-\eta}\right] h \mathbb{E}\left|\widehat{\varepsilon}_{n}\right|^{2}+8\left(L_{f}\right)^{2} \frac{1}{1-\eta} h \mathbb{E}\left|\widehat{\varepsilon}_{n+1}\right|^{2} \\
& +\left(8\left(L_{f}\right)^{2}+4\left(L_{\bar{u}}\right)^{2}\right)(1+\eta) h \mathbb{E}\left|\varepsilon_{n-m}\right|^{2}+8\left(L_{f}\right)^{2}(1+\eta) h \mathbb{E}\left|\varepsilon_{n+1-m}\right|^{2} \\
& \leq\left[1+\left(8\left(L_{f}\right)^{2}+4\left(L_{\bar{u}}\right)^{2}\right) \max \left\{\frac{1}{1-\eta}, 1+\eta\right\}\right] h, \\
& {\left[\mathbb{E}\left|\widehat{\varepsilon}_{n}\right|^{2}+\mathbb{E}\left|\widehat{\varepsilon}_{n+1}\right|^{2}+\mathbb{E}\left|\varepsilon_{n+1-m}\right|^{2}+\mathbb{E}\left|\varepsilon_{n-m}\right|^{2}\right]} \\
& =J_{6} h\left[\mathbb{E}\left|\widehat{\varepsilon}_{n}\right|^{2}+\mathbb{E}\left|\widehat{\varepsilon}_{n+1}\right|^{2}+\mathbb{E}\left|\varepsilon_{n+1-m}\right|^{2}+\mathbb{E}\left|\varepsilon_{n-m}\right|^{2}\right],
\end{aligned}
$$


where $J_{6}=1+\left(8\left(L_{f}\right)^{2}+4\left(L_{\bar{u}}\right)^{2}\right) \max \{1 /(1-\eta), 1+\eta\}$. Inserting (3.18), (3.20), (3.22), and (3.24) into (3.17) yields

$$
\begin{aligned}
\mathbb{E}\left|\widehat{\varepsilon}_{n+1}\right|^{2} \leq & \mathbb{E}\left|\widehat{\varepsilon}_{n}\right|^{2}+2 J_{2} h^{2 p_{2}}+2 J_{3} h\left(\mathbb{E}\left|\widehat{\varepsilon}_{n}\right|^{2}+\mathbb{E}\left|\varepsilon_{n-m}\right|^{2}+\mathbb{E}\left|\widehat{\varepsilon}_{n+1}\right|^{2}+\mathbb{E}\left|\varepsilon_{n+1-m}\right|^{2}\right) \\
& +h \mathbb{E}\left|\widehat{\varepsilon}_{n}\right|^{2}+J_{5} h^{2 p_{1}-1}+J_{6} h\left(\mathbb{E}\left|\widehat{\varepsilon}_{n}\right|^{2}+\mathbb{E}\left|\widehat{\varepsilon}_{n+1}\right|^{2}+\mathbb{E}\left|\varepsilon_{n+1-m}\right|^{2}+\mathbb{E}\left|\varepsilon_{n-m}\right|^{2}\right) \\
= & \left(2 J_{3}+J_{6}\right) h \mathbb{E}\left|\widehat{\varepsilon}_{n+1}\right|^{2}+\left(1+\left(1+2 J_{3}+J_{6}\right) h\right) \mathbb{E}\left|\widehat{\varepsilon}_{n}\right|^{2} \\
& +\left(2 J_{3}+J_{6}\right) h\left(\mathbb{E}\left|\varepsilon_{n+1-m}\right|^{2}+\mathbb{E}\left|\varepsilon_{n-m}\right|^{2}\right)+\left(2 J_{2}+J_{5}\right) h^{2 p_{2}} .
\end{aligned}
$$

The following proof is analogous to that of Theorem 3.1 in [5]; thus, it is not hard to derive the convergence result. The proof is completed.

Remark 3.6. Notice that if $u(\cdot)=0$ in (2.1), then (2.1) reduces to the NSDDEs without jumps, our Theorem 3.5 coincides with Theorem 3.1 in [5], that is to say, Theorem 3.5 generalizes Theorem 3.1 in [5] to the case of NSDDEs with jumps.

\section{The Examples}

Theorem 3.5 presents the convergence result about the general implicit one-step methods for NSDDEs with jumps. In this section, we discuss the applicability of the theory presented in the previous section. We will give the convergence orders of the stochastic $\theta$-methods and the balanced implicit methods.

Example 4.1. Consider the stochastic $\theta$-methods for system (2.1),

$$
\begin{aligned}
Y_{n+1}= & G\left(Y_{n+1-m}\right)+Y_{n}-G\left(Y_{n-m}\right) \\
& +\left[(1-\theta) f\left(Y_{n}, Y_{n-m}\right)+\theta f\left(Y_{n+1}, Y_{n+1-m}\right)\right] h \\
& +g\left(Y_{n}, Y_{n-m}\right) \Delta W_{n}+u\left(Y_{n}, Y_{n-m}\right) \Delta N_{n}, \quad n=0,1, \ldots, q-1,
\end{aligned}
$$

where $m=\tau / h, 0 \leq \theta \leq 1$.

Lemma 4.2. Let Assumption 1 hold, then the stochastic $\theta$-methods (4.1) are consistent with order $p_{1}=3 / 2$ in the mean and order $p_{2}=1$ in the mean square sense.

Proof. Combining (2.1), (3.2) with (4.1) yields

$$
\begin{aligned}
\delta_{n+1}= & x\left(t_{n+1}\right)-\bar{Y}\left(t_{n+1}\right) \\
= & \int_{t_{n}}^{t_{n+1}}\left[f(x(s), x(s-\tau))-f\left(x\left(t_{n}\right), x\left(t_{n}-\tau\right)\right)\right] d s \\
& +\int_{t_{n}}^{t_{n+1}}\left[g(x(s), x(s-\tau))-g\left(x\left(t_{n}\right), x\left(t_{n}-\tau\right)\right)\right] d W(s)
\end{aligned}
$$




$$
\begin{aligned}
& +\int_{t_{n}}^{t_{n+1}}\left[u(x(s), x(s-\tau))-u\left(x\left(t_{n}\right), x\left(t_{n}-\tau\right)\right)\right] d N(s) \\
& -\theta h\left[f\left(x\left(t_{n+1}\right), x\left(t_{n+1}-\tau\right)\right)-f\left(x\left(t_{n}\right), x\left(t_{n}-\tau\right)\right)\right] .
\end{aligned}
$$

Noticing the compensated Poisson process, $\widetilde{N}(t)=N(t)-\lambda t$, which satisfies

$$
\mathbb{E}\left(\int_{a}^{b} u(s) d \widetilde{N}(s) \mid \mathscr{F}_{a}\right)=0
$$

Using Assumption 1, (4.3), Hölder inequality, Lemma 2.4, the properties of conditional expectation, and Jenson's inequality: $|\mathbb{E}(x \mid \mathcal{F})|^{2} \leq \mathbb{E}\left(|x|^{2} \mid \mathcal{F}\right)$, we compute that

$$
\begin{aligned}
\mathbb{E}\left|\mathbb{E}\left(\delta_{n+1} \mid \mathcal{F}_{t_{n}}\right)\right|^{2} \leq & 5 \mathbb{E}\left|\mathbb{E}\left(\int_{t_{n}}^{t_{n+1}}\left[f(x(s), x(s-\tau))-f\left(x\left(t_{n}\right), x\left(t_{n}-\tau\right)\right)\right] d s \mid \mathcal{F}_{t_{n}}\right)\right|^{2} \\
& +5 \mathbb{E}\left|\mathbb{E}\left(\int_{t_{n}}^{t_{n+1}}\left[g(x(s), x(s-\tau))-g\left(x\left(t_{n}\right), x\left(t_{n}-\tau\right)\right)\right] d W(s) \mid \mathcal{F}_{t_{n}}\right)\right|^{2} \\
& +5 \mathbb{E}\left|\mathbb{E}\left(\int_{t_{n}}^{t_{n+1}}\left[u(x(s), x(s-\tau))-u\left(x\left(t_{n}\right), x\left(t_{n}-\tau\right)\right)\right] d \widetilde{N}(s) \mid \mathcal{F}_{t_{n}}\right)\right|^{2} \\
& +5 \lambda^{2} \mathbb{E}\left|\mathbb{E}\left(\int_{t_{n}}^{t_{n+1}}\left[u(x(s), x(s-\tau))-u\left(x\left(t_{n}\right), x\left(t_{n}-\tau\right)\right)\right] d s \mid \mathcal{F}_{t_{n}}\right)\right|^{2} \\
& +5 h^{2} \mathbb{E}\left|\mathbb{E}\left(f\left(x\left(t_{n+1}\right), x\left(t_{n+1}-\tau\right)\right)-f\left(x\left(t_{n}\right), x\left(t_{n}-\tau\right)\right) \mid \mathcal{F}_{t_{n}}\right)\right|^{2} \\
\leq & 5 \mathbb{E}\left[\mathbb{E}\left(\left|\int_{t_{n}}^{t_{n+1}} f(x(s), x(s-\tau))-f\left(x\left(t_{n}\right), x\left(t_{n}-\tau\right)\right) d s\right|^{2} \mid \mathcal{F}_{t_{n}}\right)\right] \\
& +5 \lambda^{2} \mathbb{E}\left[\mathbb{E}\left(\left.\left|\int_{t_{n}}^{t_{n+1}} u(x(s), x(s-\tau))-u\left(x\left(t_{n}\right), x\left(t_{n}-\tau\right)\right) d s\right|\right|^{2} \mid \mathcal{F}_{t_{n}}\right)\right]^{2} \\
& +5 h^{2} \mathbb{E}\left[\mathbb{E}\left(\left|f\left(x\left(t_{n+1}\right), x\left(t_{n+1}-\tau\right)\right)-f\left(x\left(t_{n}\right), x\left(t_{n}-\tau\right)\right)\right|^{2} \mid \mathcal{F}_{t_{n}}\right)\right] \\
\leq & 5\left(1+\lambda^{2}\right) K h \int_{t_{n}}^{t_{n+1}} \mathbb{E}\left(\left|x(s)-x\left(t_{n}\right)\right|^{2}+\left|x(s-\tau)-x\left(t_{n}-\tau\right)\right|^{2}\right) d s
\end{aligned}
$$




$$
\begin{aligned}
& +5 h^{2} K \mathbb{E}\left(\left|x\left(t_{n+1}\right)-x\left(t_{n}\right)\right|^{2}+\left|x\left(t_{n+1}-\tau\right)-x\left(t_{n}-\tau\right)\right|^{2}\right) \\
\leq & {\left[10\left(1+\lambda^{2}\right) K \widehat{H}+10 K \widehat{H}\right] h^{3} } \\
\mathbb{E}\left|\delta_{n+1}\right|^{2} \leq & 4 h \mathbb{E} \int_{t_{n}}^{t_{n+1}}\left|f(x(s), x(s-\tau))-f\left(x\left(t_{n}\right), x\left(t_{n}-\tau\right)\right)\right|^{2} d s \\
& +4 \mathbb{E} \int_{t_{n}}^{t_{n+1}}\left|g(x(s), x(s-\tau))-g\left(x\left(t_{n}\right), x\left(t_{n}-\tau\right)\right)\right|^{2} d s \\
& +8 \lambda \int_{t_{n}}^{t_{n+1}} \mathbb{E}\left|u(x(s), x(s-\tau))-u\left(x\left(t_{n}\right), x\left(t_{n}-\tau\right)\right)\right|^{2} d s \\
& +8 \lambda^{2} h \int_{t_{n}}^{t_{n+1}} \mathbb{E}\left|u(x(s), x(s-\tau))-u\left(x\left(t_{n}\right), x\left(t_{n}-\tau\right)\right)\right|^{2} d s \\
& +4 h^{2} \mathbb{E}\left|f\left(x\left(t_{n+1}\right), x\left(t_{n+1}-\tau\right)\right)-f\left(x\left(t_{n}\right), x\left(t_{n}-\tau\right)\right)\right|^{2} \\
\leq & \left(4 h+4+8 \lambda+8 \lambda^{2} h\right) K \int_{t_{n}}^{t_{n+1}} \mathbb{E}\left(\left|x(s)-x\left(t_{n}\right)\right|^{2}+\left|x(s-\tau)-x\left(t_{n}-\tau\right)\right|^{2}\right) d s \\
\leq & 8 K \widehat{H}\left(1+2 T+2 \lambda+2 \lambda^{2} T\right) h^{2}, \\
& +4 K h^{2} \mathbb{E}\left(\left|x\left(t_{n+1}\right)-x\left(t_{n}\right)\right|^{2}+\left|x\left(t_{n+1}-\tau\right)-x\left(t_{n}-\tau\right)\right|^{2}\right) \\
&
\end{aligned}
$$

where the compensated Poisson process $\widetilde{N}(t)=N(t)-\lambda t$ satisfies

$$
\mathbb{E}\left|\int_{t_{1}}^{t_{2}} u(x(s)) d \widetilde{N}(s)\right|^{2}=\lambda \int_{t_{1}}^{t_{2}} \mathbb{E}|u(x(s))|^{2} d s .
$$

Hence, the stochastic $\theta$-methods (4.1) are consistent with order $3 / 2$ in the mean and order 1 in the mean square sense. The proof is completed.

Theorem 4.3. Let Assumption 1 hold, then the stochastic $\theta$-methods (4.1) are convergent with order $p=1 / 2$ in the mean square sense.

Proof. Lemma 4.2 shows that the stochastic $\theta$-methods (4.1) are consistent with order $p_{1}=3 / 2$ in the mean and order $p_{2}=1$ in the mean square sense. In order to prove that the stochastic $\theta$-methods (4.1) are convergent with order $p=1 / 2$ in the mean square sense, by Theorem 3.5, we only need to verify the conditions (3.7)-(3.11). 
From (4.1), we find that

$$
\begin{gathered}
\Phi_{f}\left(h, Y_{n}, Y_{n+1}, Y_{n-m}, Y_{n+1-m}, \Delta W_{n}, \Delta N_{n}\right)=\left[(1-\theta) f\left(Y_{n}, Y_{n-m}\right)+\theta f\left(Y_{n+1}, Y_{n+1-m}\right)\right] h, \\
\Phi_{g}\left(h, Y_{n}, Y_{n-m}, \Delta W_{n}, \Delta N_{n}\right)=g\left(Y_{n}, Y_{n-m}\right) \Delta W_{n}, \\
\Phi_{u}\left(h, Y_{n}, Y_{n-m}, \Delta W_{n}, \Delta N_{n}\right)=u\left(Y_{n}, Y_{n-m}\right) \Delta N_{n} .
\end{gathered}
$$

For the random variables $x_{i}, y_{i} \in \mathbb{R}^{d}(i=1,2,3,4)$, by Assumption 1 , we have

$$
\begin{aligned}
& \left|\Phi_{f}\left(h, x_{1}, x_{2}, x_{3}, x_{4}, \Delta W_{n}, \Delta N_{n}\right)-\Phi_{f}\left(h, y_{1}, y_{2}, y_{3}, y_{4}, \Delta W_{n}, \Delta N_{n}\right)\right| \\
& \quad=\left|\left[(1-\theta) f\left(x_{1}, x_{3}\right)+\theta f\left(x_{2}, x_{4}\right)\right] h-\left[(1-\theta) f\left(y_{1}, y_{3}\right)+\theta f\left(y_{2}, y_{4}\right)\right] h\right| \\
& \quad \leq \sqrt{K} h \sum_{i=1}^{4}\left|x_{i}-y_{i}\right| .
\end{aligned}
$$

Noticing that $\mathbb{E}\left|\Delta W_{n}\right|^{2}=b h, \mathbb{E}\left|\Delta N_{n}\right|^{2}=\lambda h(1+\lambda h)$, and the random variables $x_{1}, x_{3}, y_{1}, y_{3}$ are $\mathcal{F}_{t_{n}}$-measurable, we derive that

$$
\begin{aligned}
& \mathbb{E} \mid \Phi_{g}\left(h, x_{1}, x_{3}, \Delta W_{n}, \Delta N_{n}\right)-\left.\Phi_{g}\left(h, y_{1}, y_{3}, \Delta W_{n}, \Delta N_{n}\right)\right|^{2} \\
& \quad \leq \mathbb{E}\left|g\left(x_{1}, x_{3}\right) \Delta W_{n}-g\left(y_{1}, y_{3}\right) \Delta W_{n}\right|^{2} \\
& \quad \leq b \mathrm{~K} h \mathbb{E}\left|x_{1}-y_{1}\right|^{2}+\mathbb{E}\left|x_{3}-y_{3}\right|^{2}, \\
& \mathbb{E}\left|\Phi_{u}\left(h, x_{1}, x_{3}, \Delta W_{n}, \Delta N_{n}\right)-\Phi_{u}\left(h, y_{1}, y_{3}, \Delta W_{n}, \Delta N_{n}\right)\right|^{2} \\
& \quad \leq \mathbb{E}\left|u\left(x_{1}, x_{3}\right) \Delta N_{n}-u\left(y_{1}, y_{3}\right) \Delta N_{n}\right|^{2} \\
& \quad \leq \lambda(1+\lambda T) K h\left[\mathbb{E}\left|x_{1}-y_{1}\right|^{2}+\mathbb{E}\left|x_{3}-y_{3}\right|^{2}\right] .
\end{aligned}
$$

From (4.8)-(4.10), we see that the increment functions of the stochastic $\theta$-methods (4.1) satisfy the inequalities (3.7)-(3.9) with $L_{f}=\sqrt{K}, L_{g}=b K$, and $L_{u}=\lambda(1+\lambda T) K$. Noting that $\Delta W_{n}, \Delta N_{n}$ are independent of $\mathcal{F}_{t_{n}}$ and $x_{1}, x_{3}, y_{1}$, and $y_{3}$ are $\mathcal{F}_{t_{n}}$-measurable, then using $|\mathbb{E}(x \mid \mathcal{F})| \leq \mathbb{E}(|x| \mid \mathcal{F})$, we find that

$$
\begin{aligned}
& \mathbb{E}\left[\Phi_{g}\right.\left.\left(h, x_{1}, x_{3}, \Delta W_{n}, \Delta N_{n}\right)-\Phi_{g}\left(h, y_{1}, y_{3}, \Delta W_{n}, \Delta N_{n}\right) \mid \mathcal{F}_{t_{n}}\right] \\
&= \mathbb{E}\left[g\left(x_{1}, x_{3}\right) \Delta W_{n}-g\left(y_{1}, y_{3}\right) \Delta W_{n} \mid \mathcal{F}_{t_{n}}\right] \\
& \quad= {\left[g\left(x_{1}, x_{3}\right)-g\left(y_{1}, y_{3}\right)\right] \mathbb{E}\left(\Delta W_{n} \mid \mathcal{F}_{t_{n}}\right)=0, } \\
&\left|\mathbb{E}\left[\Phi_{u}\left(h, x_{1}, x_{3}, \Delta W_{n}, \Delta N_{n}\right)-\Phi_{u}\left(h, y_{1}, y_{3}, \Delta W_{n}, \Delta N_{n}\right) \mid \mathcal{F}_{n}\right]\right| \\
&=\left|\mathbb{E}\left[u\left(x_{1}, x_{3}\right) \Delta N_{n}-u\left(y_{1}, y_{3}\right) \Delta N_{n} \mid \mathcal{F}_{t_{n}}\right]\right| \\
&=\left|\left[u\left(x_{1}, x_{3}\right)-u\left(y_{1}, y_{3}\right)\right]\right| \cdot \mathbb{E}\left(\Delta N_{n} \mid \mathcal{F}_{t_{n}}\right) \\
& \leq \lambda \sqrt{K} h\left[\left|x_{1}-y_{1}\right|+\left|x_{3}-y_{3}\right|\right] .
\end{aligned}
$$


Here, the fact used has been that $\mathbb{E} \Delta W_{n}=0$ and $\mathbb{E} \Delta N_{n}=\lambda h$. From above, we find that the increment functions of the stochastic $\theta$-methods (4.1) satisfy the estimations (3.10) and (3.11) with $L_{\bar{u}}=\lambda \sqrt{K}$. Therefore, the conditions (3.7)-(3.11) are satisfied. By Lemma 4.2 and Theorem 3.5, it is not difficult to find that the stochastic $\theta$-methods (4.1) are convergent with order $p=1 / 2$ in the mean square sense. The proof is completed.

Remark 4.4. For the case of $G(\cdot)=0,(2.1)$ reduces to the stochastic delay differential equations with jumps

$$
\begin{aligned}
\mathrm{d} x(t)= & f\left(x\left(t^{-}\right), x\left(t^{-}-\tau\right)\right) \mathrm{d} t+g\left(x\left(t^{-}\right), x\left(t^{-}-\tau\right)\right) \mathrm{d} W(t) \\
& +u\left(x\left(t^{-}\right), x\left(t^{-}-\tau\right)\right) \mathrm{d} N(t), \quad 0<t<T, \\
x(t)= & \psi(t), \quad-\tau \leq t \leq 0,
\end{aligned}
$$

where $\psi \in L_{\mp_{0}}^{2}\left([-\tau, 0] ; \mathbb{R}^{d}\right)$.

Theorem 4.3 implies that the stochastic $\theta$-methods for (4.12) are convergent with order $p=1 / 2$, which coincides with Theorem 3.2 in [11].

Example 4.5. Consider the balanced implicit methods for system (2.1),

$$
\begin{aligned}
Y_{n+1}= & G\left(Y_{n+1-m}\right)+Y_{n}-G\left(Y_{n-m}\right)+f\left(Y_{n}, Y_{n-m}\right) h \\
& +g\left(Y_{n}, Y_{n-m}\right) \Delta W_{n}+u\left(Y_{n}, Y_{n-m}\right) \Delta N_{n} \\
& +C\left(Y_{n}, Y_{n-m}\right)\left[Y_{n}-G\left(Y_{n-m}\right)-Y_{n+1}+G\left(Y_{n+1-m}\right)\right] .
\end{aligned}
$$

Here, the $d \times d$ matrix $C\left(Y_{n}, Y_{n-m}\right)$ is given by

$$
C\left(Y_{n}, Y_{n-m}\right)=C_{0}\left(Y_{n}, Y_{n-m}\right) h+C_{1}\left(Y_{n}, Y_{n-m}\right)\left|\Delta W_{n}\right|=C_{0 n} h+C_{1 n}\left|\Delta W_{n}\right|
$$

where the $C_{0 n}=C_{0}\left(Y_{n}, Y_{n-m}\right), C_{1 n}=C_{1}\left(Y_{n}, Y_{n-m}\right)$ are, in general matrix, called control functions which are often chosen as constants.

Furthermore, the control functions must satisfy some conditions.

Assumption 4. The $C_{0}(x, y)$ and $C_{1}(x, y)$ represent bounded $d \times d$-matrix-valued functions for $x, y \in \mathbb{R}^{d}$. For any real numbers $\alpha_{0} \in[0, \bar{\alpha}], \alpha_{1} \geq 0$, where $\bar{\alpha} \geq h$ for all step sizes $h$ considered and $x, y \in \mathbb{R}^{d}$, the matrix $M(x, y)=I+\alpha_{0} C_{0}(x, y)+\alpha_{1} C_{1}(x, y)$ has an inverse and satisfies the condition

$$
\left|(M(x, y))^{-1}\right| \leq H<\infty
$$


Here, $I$ is the unit matrix, and $H$ is a positive constant. Notice that if $C_{0 n}, C_{1 n}$ satisfy Assumption 4, the methods (4.13) are well defined and can be rewritten as

$$
\begin{aligned}
Y_{n+1}= & G\left(Y_{n+1-m}\right)+Y_{n}-G\left(Y_{n-m}\right)+\left[I+C\left(Y_{n}, Y_{n-m}\right)\right]^{-1} \\
& \times\left(f\left(Y_{n}, Y_{n-m}\right) h+g\left(Y_{n}, Y_{n-m}\right) \Delta W_{n}+u\left(Y_{n}, Y_{n-m}\right) \Delta N_{n}\right) .
\end{aligned}
$$

Lemma 4.6 (see [12]). If $F(y, \omega)$ is independent of $\mathcal{F}, y \in \mathbb{R}^{d}, \omega \in \Omega, \mathbb{E}(F(y, \omega))=\varphi(y)$, and $\varsigma$ is $\mathcal{F}$-measurable, then $\mathbb{E}(F(\varsigma, \omega) \mid \mp)=\varphi(\varsigma)$.

For simplicity, from now on, we suppose that $C_{0 n}, C_{1 n}$ in (4.14) are constants, that is to say, $C_{0 n}=C_{0}, C_{1 n}=C_{1}$.

Lemma 4.7. Let Assumptions 1, 2, and 4 hold, then the balanced implicit methods (4.13) are consistent with order $p_{1}=3 / 2$ in the mean and order $p_{2}=1$ in the mean square sense.

Proof. Without loss of generality, we can assume that $0<h<1$. From Lemma 4.2, we know that the Euler-Maruyama method is consistent with order 3/2 in the mean, thus using (4.4), we have

$$
\begin{aligned}
\mathbb{E}\left[\left|\mathbb{E}\left[x\left(t_{n+1}\right)-\bar{Y}^{B}\left(t_{n+1}\right) \mid \mathcal{F}_{t_{n}}\right]\right|^{2}\right] \\
=\mathbb{E}\left\{\left|\mathbb{E}\left[x\left(t_{n+1}\right)-\bar{Y}^{E}\left(t_{n+1}\right) \mid \mathcal{F}_{t_{n}}\right]+\mathbb{E}\left[\bar{Y}^{E}\left(t_{n+1}\right)-\bar{Y}^{B}\left(t_{n+1}\right) \mid \mathcal{F}_{t_{n}}\right]\right|^{2}\right\} \\
\leq 2 \mathbb{E}\left[\left|\mathbb{E}\left[x\left(t_{n+1}\right)-\bar{Y}^{E}\left(t_{n+1}\right) \mid \mathcal{F}_{t_{n}}\right]\right|^{2}\right]+2 \mathbb{E}\left[\left|\mathbb{E}\left[\bar{Y}^{E}\left(t_{n+1}\right)-\bar{Y}^{B}\left(t_{n+1}\right) \mid \mathcal{F}_{t_{n}}\right]\right|^{2}\right] \\
\leq\left[20\left(1+\lambda^{2}\right) K \widehat{H}+20 K \widehat{H}\right] h^{3}+2 \mathbb{E}\left[\left|\mathbb{E}\left[\bar{Y}^{E}\left(t_{n+1}\right)-\bar{Y}^{B}\left(t_{n+1}\right) \mid \mathcal{F}_{t_{n}}\right]\right|^{2}\right],
\end{aligned}
$$

where $\bar{Y}^{E}\left(t_{n+1}\right)$ and $\bar{Y}^{B}\left(t_{n+1}\right)$ are defined as follows:

$$
\begin{aligned}
\bar{Y}^{E}\left(t_{n+1}\right)= & G\left(x\left(t_{n+1}-\tau\right)\right)+x\left(t_{n}\right)-G\left(x\left(t_{n}-\tau\right)\right)+f\left(x\left(t_{n}\right), x\left(t_{n}-\tau\right)\right) h \\
& +g\left(x\left(t_{n}\right), x\left(t_{n}-\tau\right)\right) \Delta W_{n}+u\left(x\left(t_{n}\right), x\left(t_{n}-\tau\right)\right) \Delta N_{n}, \\
\bar{Y}^{B}\left(t_{n+1}\right)= & G\left(x\left(t_{n+1}-\tau\right)\right)+x\left(t_{n}\right)-G\left(x\left(t_{n}-\tau\right)\right)+\left[I+C_{n}\right]^{-1} \\
& \times\left(f\left(x\left(t_{n}\right), x\left(t_{n}-\tau\right)\right) h+g\left(x\left(t_{n}\right), x\left(t_{n}-\tau\right)\right) \Delta W_{n}+u\left(x\left(t_{n}\right), x\left(t_{n}-\tau\right)\right) \Delta N_{n}\right),
\end{aligned}
$$


where $C_{n}=C_{0} h+C_{1}\left|\Delta W_{n}\right|$. By (4.18), we have

$$
\begin{aligned}
\bar{Y}^{E}\left(t_{n+1}\right)-\bar{Y}^{B}\left(t_{n+1}\right)= & \left(I-\left[I+C_{n}\right]^{-1}\right) \\
& \cdot\left(f\left(x\left(t_{n}\right), x\left(t_{n}-\tau\right)\right) h+g\left(x\left(t_{n}\right), x\left(t_{n}-\tau\right)\right) \Delta W_{n}+u\left(x\left(t_{n}\right), x\left(t_{n}-\tau\right)\right) \Delta N_{n}\right) \\
= & {\left[I+C_{n}\right]^{-1} \cdot\left(\left(I+C_{n}\right)-I\right) } \\
& \cdot\left(f\left(x\left(t_{n}\right), x\left(t_{n}-\tau\right)\right) h+g\left(x\left(t_{n}\right), x\left(t_{n}-\tau\right)\right) \Delta W_{n}+u\left(x\left(t_{n}\right), x\left(t_{n}-\tau\right)\right) \Delta N_{n}\right) \\
= & {\left[I+C_{n}\right]^{-1} \cdot C_{n} } \\
& \cdot\left(f\left(x\left(t_{n}\right), x\left(t_{n}-\tau\right)\right) h+g\left(x\left(t_{n}\right), x\left(t_{n}-\tau\right)\right) \Delta W_{n}+u\left(x\left(t_{n}\right), x\left(t_{n}-\tau\right)\right) \Delta N_{n}\right) .
\end{aligned}
$$

Noticing that $x\left(t_{n}\right), x\left(t_{n}-\tau\right)$ are $\mathcal{F}_{t_{n}}$-measurable, $\Delta W_{n}$ is independent of $\mathcal{F}_{t_{n}}$, and using Lemma 4.6, we find that

$$
\mathbb{E}\left[\left(I+C_{n}\right)^{-1} \cdot C_{n} \cdot g\left(x\left(t_{n}\right), x\left(t_{n}-\tau\right)\right) \Delta W_{n} \mid \mathcal{F}_{t_{n}}\right]=0
$$

We notice that $C_{0}$ and $C_{1}$ are constants; thus, there exists a positive constant $B$, such that $\left|C_{i}\right| \leq B(i=0,1)$. Since $\Delta W_{n}, \Delta N_{n}$ are independent of $\mathcal{F}_{t_{n}}$, by Assumption 4, (4.19), (4.20), $|\mathbb{E}(x \mid \mathcal{F})| \leq \mathbb{E}(|x| \mid \mathcal{F}), \mathbb{E}\left|\Delta W_{n}\right| \leq \sqrt{b h}$, and $\mathbb{E} \Delta N_{n}=\lambda h$, we obtain

$$
\begin{aligned}
&\left|\mathbb{E}\left[\bar{Y}^{E}\left(t_{n+1}\right)-\bar{Y}^{B}\left(t_{n+1}\right) \mid \mathcal{F}_{t_{n}}\right]\right| \\
& \leq\left|f\left(x\left(t_{n}\right), x\left(t_{n}-\tau\right)\right)\right| h \cdot \mathbb{E}\left(\left|\left(I+C_{n}\right)^{-1}\right| \cdot\left|C_{0} h+C_{1}\right| \Delta W_{n}|| \mid \mathcal{F}_{t_{n}}\right) \\
& \quad+\left|u\left(x\left(t_{n}\right), x\left(t_{n}-\tau\right)\right)\right| \cdot \mathbb{E}\left(\left|\left(I+C_{n}\right)^{-1}\right| \cdot\left|C_{0} h+C_{1}\right| \Delta W_{n}|| \cdot \Delta N_{n} \mid \mathcal{F}_{t_{n}}\right) \\
& \leq\left|f\left(x\left(t_{n}\right), x\left(t_{n}-\tau\right)\right)\right| H h \cdot \mathbb{E}\left(\left|C_{0} h+C_{1}\right| \Delta W_{n}|| \mid \mathcal{F}_{t_{n}}\right) \\
& \quad+\left|u\left(x\left(t_{n}\right), x\left(t_{n}-\tau\right)\right)\right| H \cdot \mathbb{E}\left(\left|C_{0} h+C_{1}\right| \Delta W_{n}|| \cdot \Delta N_{n} \mid \mathcal{F}_{t_{n}}\right) \\
& \leq\left(\left|f\left(x\left(t_{n}\right), x\left(t_{n}-\tau\right)\right)\right|+\left|u\left(x\left(t_{n}\right), x\left(t_{n}-\tau\right)\right)\right| \lambda\right) H h^{3 / 2} B(\sqrt{h}+\sqrt{b}) .
\end{aligned}
$$

It follows from Assumption 2, Lemma 2.3, and (4.21) that

$$
\begin{aligned}
\mathbb{E}\left[\left|\mathbb{E}\left[\bar{Y}^{E}\left(t_{n+1}\right)-\bar{Y}^{B}\left(t_{n+1}\right) \mid \mp_{t_{n}}\right]\right|^{2}\right] \\
\quad \leq 2 H^{2} B^{2}(\sqrt{T}+\sqrt{b})^{2} h^{3}\left[\mathbb{E}\left|f\left(x\left(t_{n}\right), x\left(t_{n}-\tau\right)\right)\right|^{2}+\mathbb{E}\left|u\left(x\left(t_{n}\right), x\left(t_{n}-\tau\right)\right)\right|^{2} \lambda^{2}\right] \\
\quad \leq 2 L H^{2} B^{2}\left(1+\lambda^{2}\right)(\sqrt{T}+\sqrt{b})^{2}\left(1+2 J_{1}\right) h^{3} .
\end{aligned}
$$


Inserting (4.22) into (4.17) yields

$$
\begin{aligned}
\mathbb{E}[\mid \mathbb{E} & {\left.\left.\left[x\left(t_{n+1}\right)-\bar{Y}^{B}\left(t_{n+1}\right) \mid \mathcal{F}_{t_{n}}\right]\right|^{2}\right] } \\
& \leq\left(20\left(1+\lambda^{2}\right) K \widehat{H}+20 K \widehat{H}+4 L H^{2} B^{2}\left(1+\lambda^{2}\right)(\sqrt{T}+\sqrt{b})^{2}\left(1+2 J_{1}\right)\right) h^{3},
\end{aligned}
$$

which implies that the balanced implicit methods (4.13) are consistent with order $3 / 2$ in the mean. In the following, we will show that the balanced implicit methods (4.13) are consistent with order 1 in the mean square sense. Using Assumptions 2 and 4, Lemma 2.3, $\left|C_{i}\right| \leq B$ $(i=0,1), \mathbb{E}\left|\Delta W_{n}\right|^{2}=b h, \mathbb{E}\left|\Delta W_{n}\right|^{4} \leq 3 b^{2} h^{2}, \mathbb{E} \Delta N_{n}^{2}=\lambda h(1+\lambda h)$, and (4.19), we compute that

$$
\begin{aligned}
& \mathbb{E}\left[\left|\bar{Y}^{E}\left(t_{n+1}\right)-\bar{Y}^{B}\left(t_{n+1}\right)\right|^{2}\right] \\
& \leq 3 h^{2} H^{2} \mathbb{E}\left(\left|C_{0} h+C_{1}\right| \Delta W_{n} \|^{2} \cdot\left|f\left(x\left(t_{n}\right), x\left(t_{n}-\tau\right)\right)\right|^{2}\right) \\
&+3 H^{2} \mathbb{E}\left(\left.\left|C_{0} h+C_{1}\right| \Delta W_{n}\right|^{2} \cdot\left|g\left(x\left(t_{n}\right), x\left(t_{n}-\tau\right)\right)\right|^{2}\left|\Delta W_{n}\right|^{2}\right) \\
&+3 H^{2} \mathbb{E}\left(\left.\left|C_{0} h+C_{1}\right| \Delta W_{n}\right|^{2} \cdot\left|u\left(x\left(t_{n}\right), x\left(t_{n}-\tau\right)\right)\right|^{2} \Delta N_{n}^{2}\right) \\
& \leq 3 h^{2} H^{2} \mathbb{E}\left(\left(2\left|C_{0}\right|^{2} h^{2}+2\left|C_{1}\right|^{2}\left|\Delta W_{n}\right|^{2}\right) \cdot\left|f\left(x\left(t_{n}\right), x\left(t_{n}-\tau\right)\right)\right|^{2}\right) \\
&+3 H^{2} \mathbb{E}\left(\left(2\left|C_{0}\right|^{2} h^{2}\left|\Delta W_{n}\right|^{2}+2\left|C_{1}\right|^{2}\left|\Delta W_{n}\right|^{4}\right) \cdot\left|g\left(x\left(t_{n}\right), x\left(t_{n}-\tau\right)\right)\right|^{2}\right) \\
&+3 H^{2} \mathbb{E}\left(\left(2\left|C_{0}\right|^{2} h^{2}+2\left|C_{1}\right|^{2}\left|\Delta W_{n}\right|^{2}\right) \cdot\left|u\left(x\left(t_{n}\right), x\left(t_{n}-\tau\right)\right)\right|^{2} \Delta N_{n}^{2}\right) \\
& \leq 3 h^{2} H^{2}\left(2 B^{2} h^{2}+2 B^{2} b h\right) \mathbb{E}\left[\left|f\left(x\left(t_{n}\right), x\left(t_{n}-\tau\right)\right)\right|^{2}\right] \\
&+3 H^{2}\left(2 B^{2} h^{2} b h+2 B^{2} \cdot 3 b^{2} h^{2}\right) \mathbb{E}\left[\left|g\left(x\left(t_{n}\right), x\left(t_{n}-\tau\right)\right)\right|^{2}\right] \\
&+3 H^{2} \lambda h(1+\lambda h)\left(2 B^{2} h^{2}+2 B^{2} b h\right) \mathbb{E}\left[\left|u\left(x\left(t_{n}\right), x\left(t_{n}-\tau\right)\right)\right|^{2}\right] \\
& \leq 6 H^{2} B^{2} L\left[T^{2}+2 b T+3 b^{2}+\lambda(1+\lambda T)(T+b)\right]\left(1+2 J_{1}\right) h^{2} .
\end{aligned}
$$

Theorem 4.3 implies that the Euler-Maruyama method is convergent with order $p=1 / 2$. Thus, by (4.5) and (4.24), we have

$$
\begin{aligned}
\mathbb{E}\left[\left|x\left(t_{n+1}\right)-\bar{Y}^{B}\left(t_{n+1}\right)\right|^{2}\right] \leq & 2 \mathbb{E}\left[\left|x\left(t_{n+1}\right)-\bar{Y}^{E}\left(t_{n+1}\right)\right|^{2}\right]+2 \mathbb{E}\left[\left|\bar{Y}^{E}\left(t_{n+1}\right)-\bar{Y}^{B}\left(t_{n+1}\right)\right|^{2}\right] \\
\leq & {\left[16 K \widehat{H}\left(1+2 T+2 \lambda+2 \lambda^{2} T\right)+12 H^{2} B^{2} L\right.} \\
& \left.\times\left[T^{2}+2 b T+3 b^{2}+\lambda(1+\lambda T)(T+b)\right]\left(1+2 J_{1}\right)\right] h^{2} .
\end{aligned}
$$

The proof is completed. 
Theorem 4.8. Let Assumptions 1-4 hold, then the balanced implicit methods (4.16) are convergent with order $p=1 / 2$ in the mean square sense.

Proof. By (4.16), the increment functions $\Phi_{f}, \Phi_{g}$, and $\Phi_{u}$ of the balanced implicit methods (4.16) are given as follows:

$$
\begin{gathered}
\Phi_{f}\left(h, Y_{n}, Y_{n+1}, Y_{n-m}, Y_{n+1-m}, \Delta W_{n}, \Delta N_{n}\right)=\left[I+C\left(Y_{n}, Y_{n-m}\right)\right]^{-1} f\left(Y_{n}, Y_{n-m}\right) h, \\
\Phi_{g}\left(h, Y_{n}, Y_{n-m}, \Delta W_{n}, \Delta N_{n}\right)=\left[I+C\left(Y_{n}, Y_{n-m}\right)\right]^{-1} g\left(Y_{n}, Y_{n-m}\right) \Delta W_{n}, \\
\Phi_{u}\left(h, Y_{n}, Y_{n-m}, \Delta W_{n}, \Delta N_{n}\right)=\left[I+C\left(Y_{n}, Y_{n-m}\right)\right]^{-1} u\left(Y_{n}, Y_{n-m}\right) \Delta N_{n} .
\end{gathered}
$$

For $x_{i}, y_{i} \in \mathbb{R}^{d}(i=1,2,3,4)$, by Assumptions 1-4, (4.26), we arrive at

$$
\begin{aligned}
& \left|\Phi_{f}\left(h, x_{1}, x_{2}, x_{3}, x_{4}, \Delta W_{n}, \Delta N_{n}\right)-\Phi_{f}\left(h, y_{1}, y_{2}, y_{3}, y_{4}, \Delta W_{n}, \Delta N_{n}\right)\right| \\
& \quad=\left|\left[I+C_{0} h+C_{1}\left|\Delta W_{n}\right|\right]^{-1} f\left(x_{1}, x_{3}\right) h-\left[I+C_{0} h+C_{1}\left|\Delta W_{n}\right|\right]^{-1} f\left(y_{1}, y_{3}\right) h\right| \\
& \quad \leq H \sqrt{K} h\left(\left|x_{1}-y_{1}\right|+\left|x_{3}-y_{3}\right|\right) .
\end{aligned}
$$

Noticing that $\mathbb{E}\left|\Delta W_{n}\right|^{2}=b h, \mathbb{E} \Delta N_{n}^{2}=\lambda h(1+\lambda h)$, the random variables $x_{1}, x_{3}, y_{1}$, and $y_{3}$ are $\mathcal{F}_{t_{n}}$-measurable and using Assumptions 1-4, (4.27), we obtain

$$
\begin{aligned}
& \mathbb{E}\left|\Phi_{g}\left(h, x_{1}, x_{3}, \Delta W_{n}, \Delta N_{n}\right)-\Phi_{g}\left(h, y_{1}, y_{3}, \Delta W_{n}, \Delta N_{n}\right)\right|^{2} \\
& \left.\quad=\mathbb{E} \mid\left[I+C_{n}\right)\right]^{-1} g\left(x_{1}, x_{3}\right) \Delta W_{n}-\left.\left[I+C_{n}\right]^{-1} g\left(y_{1}, y_{3}\right) \Delta W_{n}\right|^{2} \\
& \quad \leq H^{2} b K h\left[\mathbb{E}\left|x_{1}-y_{1}\right|^{2}+\mathbb{E}\left|x_{3}-y_{3}\right|^{2}\right] \\
& \mathbb{E}\left|\Phi_{u}\left(h, x_{1}, x_{3}, \Delta W_{n}, \Delta N_{n}\right)-\Phi_{u}\left(h, y_{1}, y_{3}, \Delta W_{n}, \Delta N_{n}\right)\right|^{2} \\
& \quad=\mathbb{E}\left|\left[I+C_{n}\right]^{-1} u\left(x_{1}, x_{3}\right) \Delta N_{n}-\left[I+C_{n}\right]^{-1} u\left(y_{1}, y_{3}\right) \Delta N_{n}\right|^{2} \\
& \quad \leq H^{2} K \lambda(1+\lambda T) h\left[\mathbb{E}\left|x_{1}-y_{1}\right|^{2}+\mathbb{E}\left|x_{3}-y_{3}\right|^{2}\right] .
\end{aligned}
$$

Since $\Delta W_{n}, \Delta N_{n}$ are independent of $\mathcal{F}_{t_{n}}$ and the random variables $x_{1}, x_{3}, y_{1}$, and $y_{3}$ are $\mathcal{F}_{t_{n}}{ }^{-}$ measurable; thus, by the inequality $|\mathbb{E}[x \mid \mathcal{F}]| \leq \mathbb{E}[|x| \mid \mathcal{F}], \mathbb{E} \Delta N_{n}=\lambda h$, and Lemma 4.6, we compute that

$$
\begin{aligned}
\mathbb{E}\left[\Phi_{g}\left(h, x_{1}, x_{3}, \Delta W_{n}, \Delta N_{n}\right)-\Phi_{g}\left(h, y_{1}, y_{3}, \Delta W_{n}, \Delta N_{n}\right) \mid \mathcal{F}_{t_{n}}\right] \\
\quad=\mathbb{E}\left[\left(I+C_{n}\right)^{-1} g\left(x_{1}, x_{3}\right) \Delta W_{n} \mid \mathcal{F}_{t_{n}}\right]-\mathbb{E}\left[\left(I+C_{n}\right)^{-1} g\left(y_{1}, y_{3}\right) \Delta W_{n} \mid \mathcal{F}_{t_{n}}\right] \\
\quad=0
\end{aligned}
$$




$$
\begin{aligned}
\left|\mathbb{E}\left[\Phi_{u}\left(h, x_{1}, x_{3}, \Delta W_{n}, \Delta N_{n}\right)-\Phi_{u}\left(h, y_{1}, y_{3}, \Delta W_{n}, \Delta N_{n}\right) \mid \mathcal{F}_{t_{n}}\right]\right| \\
\quad \leq \mathbb{E}\left|\left[I+C_{n}\right]^{-1} u\left(x_{1}, x_{3}\right) \Delta N_{n}-\left[I+C_{n}\right]^{-1} u\left(y_{1}, y_{3}\right) \Delta N_{n}\right| \mathcal{F}_{t_{n}} \mid \\
\quad \leq\left|u\left(x_{1}, x_{3}\right)-u\left(y_{1}, y_{3}\right)\right| \cdot \mathbb{E}\left[\left|\left(I+C_{n}\right)^{-1}\right| \cdot \Delta N_{n} \mid \mathcal{F}_{t_{n}}\right] \\
\quad \leq H \sqrt{K} \lambda h\left[\left|x_{1}-y_{1}\right|+\left|x_{3}-y_{3}\right|\right] .
\end{aligned}
$$

From (4.28)-(4.32), we see that the increment functions of the balanced implicit methods (4.13) satisfy the conditions (3.7)-(3.11) with $L_{f}=H \sqrt{K}, L_{g}=H^{2} K b, L_{u}=H^{2} K \lambda(1+\lambda T)$, and $L_{\bar{u}}=H \sqrt{K} \lambda$. A combination of Lemma 4.7 and Theorem 3.5 leads to the conclusion that the balanced implicit methods (4.13) are convergent with order $p=1 / 2$ in the mean square sense. The proof is completed.

Remark 4.9. For the case of $G(\cdot)=0$ and $\tau=0,(2.1)$ reduces to the stochastic differential equations with jumps

$$
\begin{gathered}
\mathrm{d} x(t)=f\left(t, x\left(t^{-}\right)\right) \mathrm{d} t+g\left(t, x\left(t^{-}\right)\right) \mathrm{d} W(t)+u\left(t, x\left(t^{-}\right)\right) \mathrm{d} N(t), \quad 0<t<T, \\
x\left(0^{-}\right)=x_{0} .
\end{gathered}
$$

From Theorem 4.8, it is not difficult to find that the balanced implicit methods for (4.33) are convergent with order $p=1 / 2$ in the mean square sense, which coincides with Theorem 2.1 in [13].

\section{Numerical Experiments}

In this section, several numerical examples are given to illustrate our theoretical results in the previous sections. Consider the nonlinear equation

$$
\begin{aligned}
\mathrm{d}[(x(t)-x(t-1))]= & {\left[-x(t)+\frac{x(t-1)}{1+x^{2}(t-1)}\right] \mathrm{d} t+[\sin (x(t))+\cos (x(t-1))] \mathrm{d} W(t) } \\
& +\frac{1}{2} x(t) \mathrm{d} N(t), \quad t \in[0, T],
\end{aligned}
$$

with initial data

$$
x(t) \equiv 1, \quad t \in[-1,0] .
$$

To show the convergence of the $\theta$-methods (4.1) and the balanced implicit methods (4.16), we choose $\theta=0.5, C_{0}=1$, and $C_{1}=0.5$. In all the numerical experiments, we identify the numerical solution using very small stepsize $h=\Delta t$ as the exact solution and compare this with the numerical approximations using $h=4 \Delta t, 8 \Delta t, 16 \Delta t, 32 \Delta t, 64 \Delta t$ for $\Delta t=2^{-14}$ over 2000 different discretized Brownian paths. The mean-square errors $\varepsilon^{r}=$ $\varepsilon_{2^{1+r} \Delta t}, r=1,2,3,4,5$, all measured at time $T=1$, are estimated by trajectory averaging, that is, 


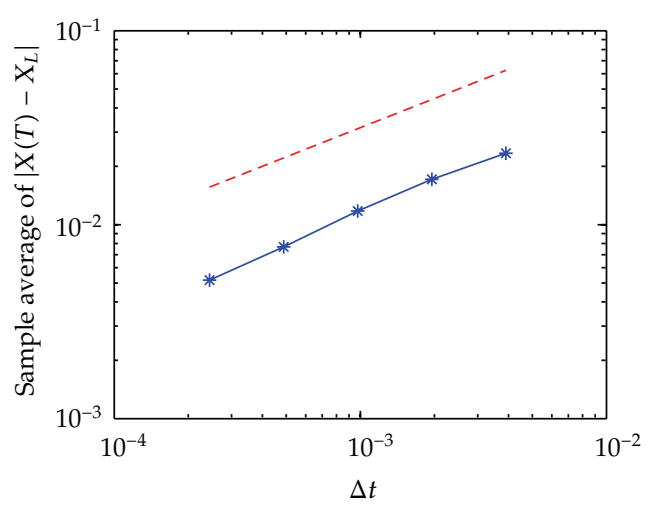

(a)

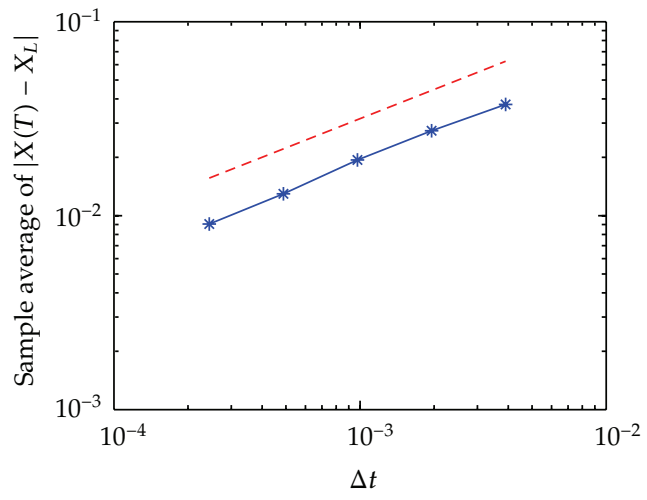

(b)

Figure 1: The convergence of the numerical methods. (a) Stochastic $\theta$-method (4.1); (b) the balanced implicit methods (4.16).

$\varepsilon^{r} \cong 1 / 2000 \sum_{j=1}^{2000}\left|x_{r, T}\left(\omega_{j}\right)-Y_{r, q}\left(\omega_{j}\right)\right|^{2}$. We plot our approximation to $\sqrt{\varepsilon^{r}}$ against $h$ on a log$\log$ scale in Figure 1. For reference, a dashed line of slope one-half is added in two graphs. In Figure 1., we show the convergence of the $\theta$-method (4.1) in the left picture and the balanced implicit methods (4.16) in the right picture, respectively.

We see that the slopes of the two curves appear to match well in two pictures in Figure 1, which is consistent with the strong order of one-half implied in Theorem 4.3 and Theorem 4.8 .

\section{Conclusion}

In this paper, we consider a family of implicit one-step methods for the NSDDEs with jumps. A relationship between the consistent order and the convergence order is established. A general framework for mean-square convergence of the methods is provided. The applicability of the mean-square convergence theory is illustrated by the stochastic $\theta$ methods and the balanced implicit methods. We have generalized the existing results. The examples presented in Section 4 show that the main result in this paper can be applied not only to semi-implicit methods but also to full implicit methods.

\section{Acknowledgments}

This research is supported with funds provided by the National Natural Science Foundation of China (no. 10871207 and 11171352) and the project sponsored by the Scientific Research Foundation for the Returned Overseas Chinese Scholars, State Education Ministry.

\section{References}

[1] G. N. Milstein, Numerical Integration of Stochastic Differential Equations, vol. 313 of Mathematics and Its Applications, Kluwer Academic Publishers Group, London, UK, 1995.

[2] P. E. Kloeden and E. Platen, Numerical Solution of Stochastic Differential Equations, vol. 23 of Applications of Mathematics (New York), Springer, Berlin, Germany, 1992.

[3] E. Buckwar, "Introduction to the numerical analysis of stochastic delay differential equations," Journal of Computational and Applied Mathematics, vol. 125, no. 1-2, pp. 297-307, 2000. 
[4] E. Buckwar, "One-step approximations for stochastic functional differential equations," Applied Numerical Mathematics, vol. 56, no. 5, pp. 667-681, 2006.

[5] H. Zhang and S. Gan, "Mean square convergence of one-step methods for neutral stochastic differential delay equations," Applied Mathematics and Computation, vol. 204, no. 2, pp. 884-890, 2008.

[6] R. Cont and P. Tankov, Financial Modelling with Jump Processes, Chapman \& Hall/CRC Financial Mathematics Series, Chapman \& Hall/CRC, Boca Raton, Fla, USA, 2004.

[7] P. Glasserman and N. Merener, "Convergence of a discretization scheme for jump-diffusion processes with state-dependent intensities," Proceedings of The Royal Society of London. Series A. Mathematical, Physical and Engineering Sciences, vol. 460, no. 2041, pp. 111-127, 2004.

[8] P. Glasserman, Monte Carlo Methods in Financial Engineering, vol. 53 of Applications of Mathematics (New York), Springer, New York, NY, USA, 2004.

[9] X. Mao, Stochastic Differential Equations and Their Applications, Horwood Publishing Series in Mathematics \& Applications, Horwood Publishing Limited, Chichester, UK, 1997.

[10] S. Gan, H. Schurz, and H. Zhang, "Mean square convergence of stochastic $\theta$-methods for nonlinear neutral stochastic differential delay equations," International Journal of Numerical Analysis and Modeling, vol. 8, no. 2, pp. 201-213, 2011.

[11] L.-s. Wang, C. Mei, and H. Xue, "The semi-implicit Euler method for stochastic differential delay equations with jumps," Applied Mathematics and Computation, vol. 192, no. 2, pp. 567-578, 2007.

[12] I. I. Gihman and A. V. Skorohod, Stokhasticheskie Differentsialnye Uravneniya, Naukova Dumka, Kyiv, Ukrania, 1968.

[13] L. Hu and S. Gan, "Convergence and stability of the balanced methods for stochastic differential equations with jumps," International Journal of Computer Mathematics, vol. 88, no. 10, pp. 2089-2108, 2011. 


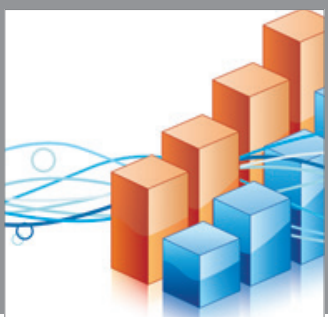

Advances in

Operations Research

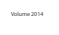

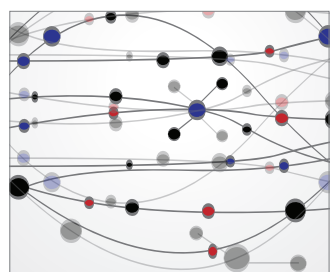

\section{The Scientific} World Journal
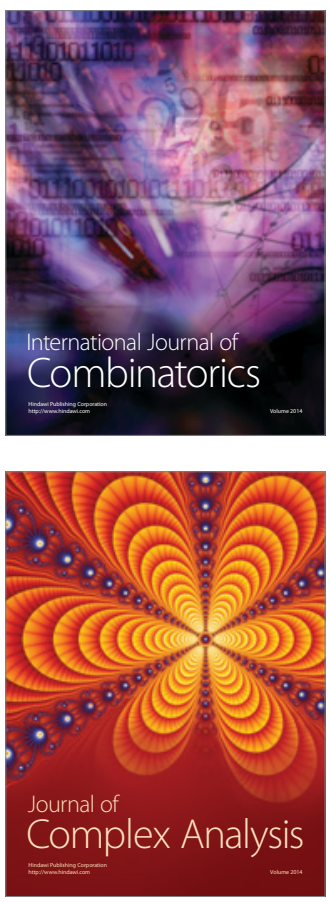

International Journal of

Mathematics and

Mathematical

Sciences
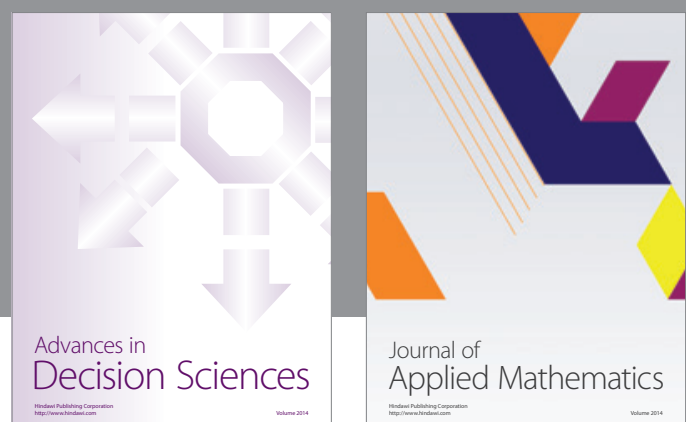

Journal of

Applied Mathematics
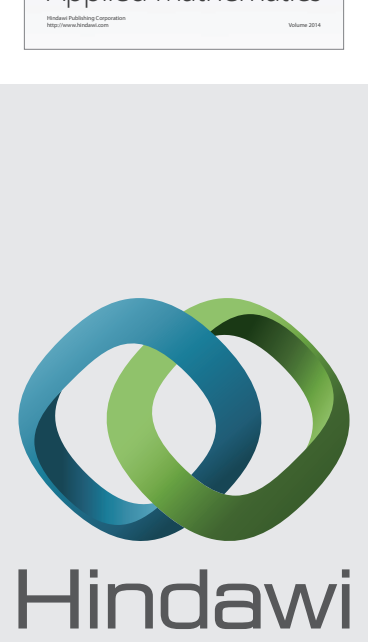

Submit your manuscripts at http://www.hindawi.com
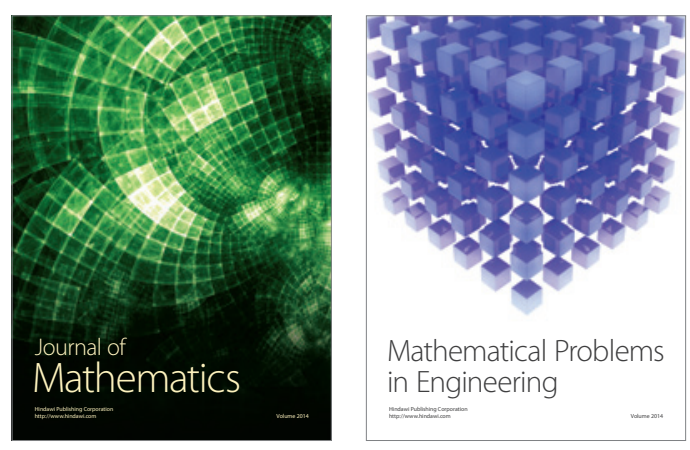

Mathematical Problems in Engineering
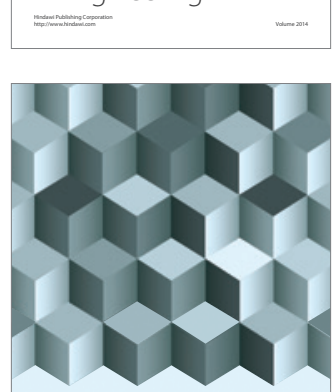

Journal of

Function Spaces
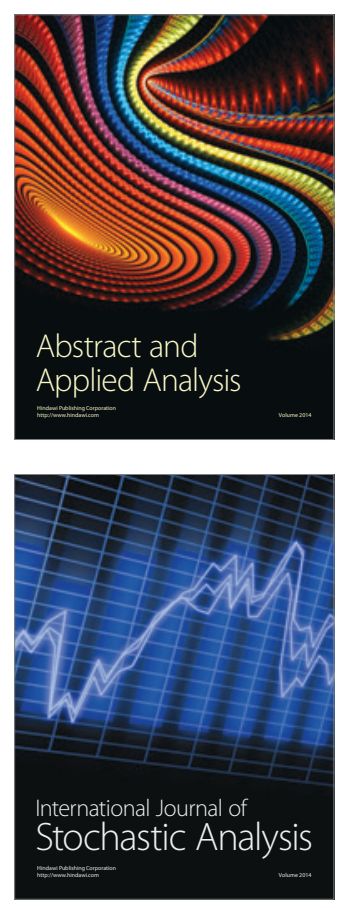

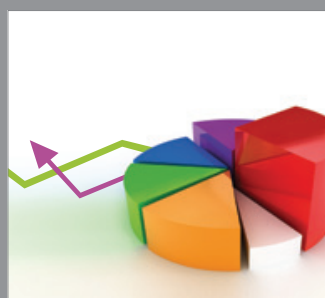

ournal of

Probability and Statistics

Promensencen
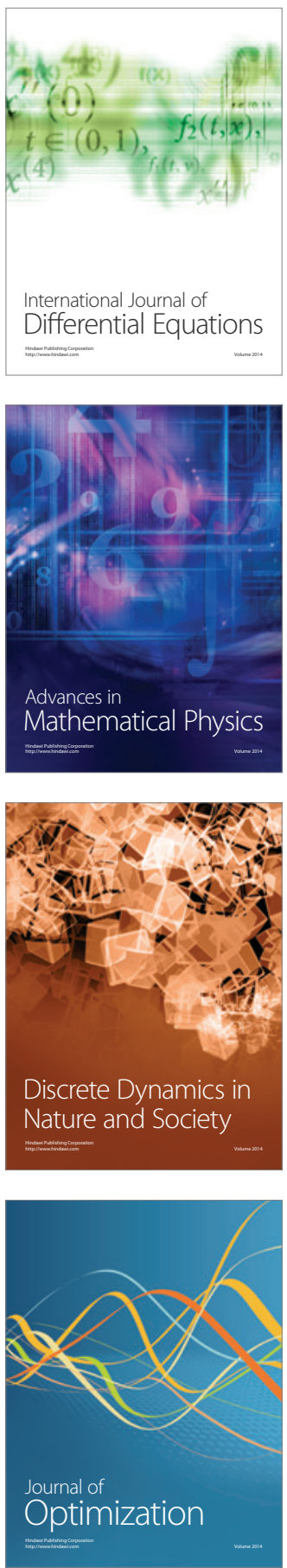